\title{
LIGO: the Laser Interferometer Gravitational-Wave Observatory
}

\section{The LIGO Scientific Collaboration, http://www.ligo.org}

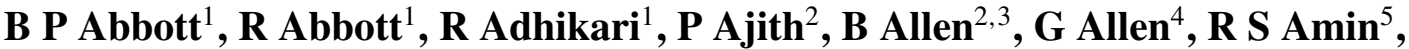
S B Anderson ${ }^{1}$, W G Anderson ${ }^{3}$, M A Arain ${ }^{6}$, M Araya ${ }^{1}$, H Armandula ${ }^{1}$,

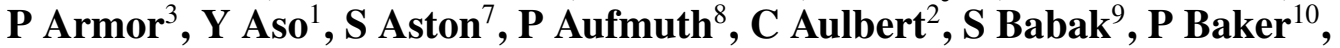
S Ballmer ${ }^{1}$, C Barker ${ }^{11}$, D Barker ${ }^{11}$, B Barr ${ }^{12}$, P Barriga ${ }^{13}$, L Barsotti ${ }^{14}$, M A Barton ${ }^{1}$, I Bartos ${ }^{15}, \mathbf{R}$ Bassiri $^{12}$, M Bastarrika ${ }^{12}$, B Behnke ${ }^{9}$, M Benacquista ${ }^{16}$, J Betzwieser $^{1}$, P T Beyersdorf ${ }^{17}$, I A Bilenko ${ }^{18}$, G Billingsley ${ }^{1}$, R Biswas ${ }^{3}$, E Black ${ }^{1}$, J K Blackburn ${ }^{1}$, L Blackburn ${ }^{14}$, D Blair ${ }^{13}$, B Bland ${ }^{11}$, T P Bodiya ${ }^{14}$, L Bogue ${ }^{19}$, R Bork ${ }^{1}$, V Boschi ${ }^{1}$, S Bose $^{20}$, P R Brady ${ }^{3}$, V B Braginsky ${ }^{18}$, J E Brau ${ }^{21}$, D O Bridges ${ }^{19}$, M Brinkmann ${ }^{2}$, A F Brooks ${ }^{1}$, D A Brown ${ }^{22}$, A Brummit ${ }^{23}$, G Brunet ${ }^{14}$, A Bullington ${ }^{4}$, A Buonanno ${ }^{24}$, O Burmeister ${ }^{2}$, R L Byer ${ }^{4}$, L Cadonati ${ }^{25}$, J B Camp ${ }^{26}$, J Cannizzo ${ }^{26}$, K C Cannon ${ }^{1}, \mathbf{J ~ C a o}^{14}, \mathbf{L}$ Cardenass $^{1}, \mathbf{S}$ Caride $^{27}, \mathbf{G}$ Castaldi $^{28}, \mathbf{S}$ Caudill ${ }^{5}$, M Cavaglià ${ }^{29}, \mathbf{C}$ Cepeda $^{1}$, T Chalermsongsak ${ }^{1}, \mathbf{E ~ C h a l k l e y ~}^{12}, \mathbf{P}$ Charlton $^{30}$, S Chatterji $^{1}$, S Chelkowski ${ }^{7}$, Y Chen ${ }^{9,31}$, N Christensen ${ }^{32}$, C T Y Chung ${ }^{33}$, D Clark ${ }^{4}$, J Clark ${ }^{34}$, J H Clayton ${ }^{3}$, T Cokelaer $^{34}$, C N Colacino ${ }^{35}$, R Conte ${ }^{36}$, D Cook $^{11}$, T R C Corbitt ${ }^{14}$, N Cornish ${ }^{10}$, D Coward ${ }^{13}$, D C Coyne ${ }^{1}$, J D E Creighton ${ }^{3}$, T D Creighton ${ }^{16}$, A M Cruise ${ }^{7}$, R M Culter ${ }^{7}$, A Cumming ${ }^{12}$, L Cunningham ${ }^{12}$, S L Danilishin ${ }^{18}$, K Danzmann ${ }^{2,8}$, B Daudert ${ }^{1}$, G Davies ${ }^{34}$, E J Daw ${ }^{37}$, D DeBra ${ }^{4}$, J Degallaix ${ }^{2}$, V Dergachev ${ }^{27}$, S Desai ${ }^{38}$, R DeSalvo ${ }^{1}$, S Dhurandhar ${ }^{39}, \mathbf{M}$ Díaz $^{16}, \mathbf{A}$ Dietz $^{34}, \mathbf{F}$ Donovan $^{14}, \mathbf{K}$ L Dooley ${ }^{6}, \mathbf{E} \mathbf{E ~ D o o m e s ~}^{40}$, R W P Drever ${ }^{41}$, J Dueck ${ }^{2}$, I Duke ${ }^{14}$, J-C Dumas ${ }^{13}$, J G Dwyer ${ }^{15}$, C Echols ${ }^{1}$, M Edgar ${ }^{12}$, A Effler ${ }^{11}$, P Ehrens ${ }^{1}$, E Espinoza ${ }^{1}$, T Etzel ${ }^{1}$, M Evans ${ }^{14}$, T Evans ${ }^{19}$,

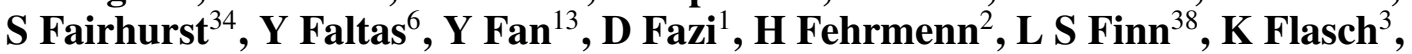
S Foley ${ }^{14}$, C Forrest ${ }^{42}$, N Fotopoulos ${ }^{3}$, A Franzen ${ }^{8}$, M Frede $^{2}$, M Frei $^{43}$, Z Frei $^{35}$,

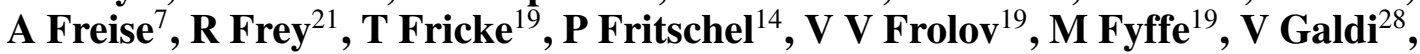
J A Garofoli ${ }^{22}$, I Gholami ${ }^{9}$, J A Giaime ${ }^{5,19}$, S Giampanis ${ }^{2}$, K D Giardina ${ }^{19}$, K Goda ${ }^{14}$, E Goetz ${ }^{27}$, L M Goggin ${ }^{3}$, G González ${ }^{5}$, M L Gorodetsky ${ }^{18}$, S Goßler ${ }^{2}$, R Gouaty ${ }^{5}$, A Grant ${ }^{12}$, S Gras ${ }^{13}$, C Gray ${ }^{11}$, M Gray ${ }^{44}$, R J S Greenhalgh ${ }^{23}$, A M Gretarsson ${ }^{45}$, F Grimaldi ${ }^{14}$, R Grosso ${ }^{16}$, H Grote $^{2}$, S Grunewald ${ }^{9}$, M Guenther ${ }^{11}$, E K Gustafson ${ }^{1}$, R Gustafson ${ }^{27}$, B Hage ${ }^{8}$, J M Hallam ${ }^{7}$,

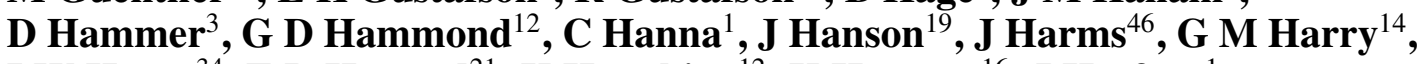
I W Harry ${ }^{34}$, E D Harstad ${ }^{21}$, K Haughian ${ }^{12}$, K Hayama ${ }^{16}$, J Heefner ${ }^{1}$, I S Heng ${ }^{12}$, A Heptonstall ${ }^{1}$, M Hewitson ${ }^{2}$, S Hild ${ }^{7}$, E Hirose ${ }^{22}$, D Hoak ${ }^{19}$, K A Hodge ${ }^{1}$, K Holt ${ }^{19}$, D J Hosken ${ }^{47}$, J Hough ${ }^{12}$, D Hoyland ${ }^{13}$, B Hughey ${ }^{14}$, S H Huttner ${ }^{12}$, D R Ingram ${ }^{11}$, T Isogai ${ }^{32}$, M Ito ${ }^{21}$, A Ivanov ${ }^{1}$, B Johnson ${ }^{11}$, W W Johnson ${ }^{5}$, D I Jones ${ }^{48}$, G Jones ${ }^{34}$, R Jones ${ }^{12}$, L Ju ${ }^{13}$, P Kalmus ${ }^{1}$, V Kalogera ${ }^{49}$, S Kandhasamy ${ }^{46}$, J Kanner ${ }^{24}$, D Kasprzyk ${ }^{7}$, E Katsavounidis ${ }^{14}$, K Kawabe $^{11}$, S Kawamura ${ }^{50}$, F Kawazoe ${ }^{2}$, W Kells ${ }^{1}$, D G Keppel ${ }^{1}$, A Khalaidovski ${ }^{2}$, F Y Khalili ${ }^{18}$, R Khan ${ }^{15}$, E Khazanov ${ }^{51}$, P King ${ }^{1}$, J S Kissel ${ }^{5}$, S Klimenko ${ }^{6}$, K Kokeyama ${ }^{50}$, V Kondrashov ${ }^{1}$, R Kopparapu $^{38}$, S Koranda ${ }^{3}$,

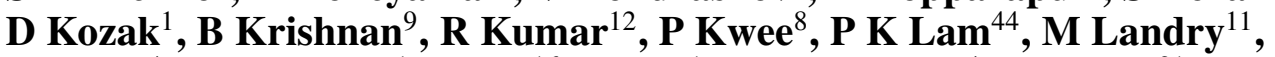
B Lantz ${ }^{4}$, A Lazzarini ${ }^{1}, \mathbf{H}$ Lei $^{16}, \mathbf{M}$ Lei $^{1}, \mathbf{N}$ Leindecker $^{4}, \mathbf{I}_{\text {Leonor }}{ }^{21}, \mathbf{C ~ L i ~}^{31}$, H Lin ${ }^{6}$, P E Lindquist ${ }^{1}$, T B Littenberg ${ }^{10}$, N A Lockerbie ${ }^{52}$, D Lodhia ${ }^{7}$, 
M Longo ${ }^{28}$, M Lormand ${ }^{19}, \mathbf{P}$ Lu $^{4}$, M Lubinski ${ }^{11}$, A Lucianetti ${ }^{6}, \mathbf{H}$ Lück $^{2,8}$, B Machenschalk ${ }^{9}$, M MacInnis ${ }^{14}$, M Mageswaran ${ }^{1}$, K Mailand $^{1}$, I Mandel ${ }^{49}$, V Mandic $^{46}$, S Márka ${ }^{15}$, Z Márka ${ }^{15}$, A Markosyan ${ }^{4}$, J Markowitz $^{14}$, E Maros ${ }^{1}$, I W Martin ${ }^{12}$, R M Martin ${ }^{6}$, J N Marx ${ }^{1}$, K Mason ${ }^{14}$, F Matichard ${ }^{5}$, L Matone ${ }^{15}$, R A Matzner ${ }^{43}$, N Mavalvala ${ }^{14}$, R McCarthy ${ }^{11}$, D E McClelland ${ }^{44}$,

S C McGuire ${ }^{40}$, M McHugh ${ }^{53}$, G McIntyre ${ }^{1}$, D J A McKechan ${ }^{34}$, K McKenzie ${ }^{44}$, M Mehmet ${ }^{2}$, A Melatos ${ }^{33}$, A C Melissinos ${ }^{42}$, D F Menéndez ${ }^{38}$, G Mendell ${ }^{11}$,

R A Mercer ${ }^{3}$, S Meshkov ${ }^{1}, \mathbf{C}$ Messenger ${ }^{2}$, M S Meyer ${ }^{19}$, J Miller ${ }^{12}$, J Minelli $^{38}$, Y Mino $^{31}$, V P Mitrofanov ${ }^{18}$, G Mitselmakher ${ }^{6}, \mathbf{R}$ Mittleman $^{14}$, O Miyakawa ${ }^{1}$,

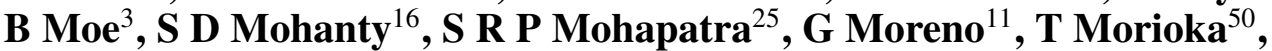
K Mors $^{2}$, K Mossavi ${ }^{2}$, C MowLowry ${ }^{44}$, G Mueller ${ }^{6}$, H Müller-Ebhardt ${ }^{2}$, D Muhammad ${ }^{19}$, S Mukherjee ${ }^{16}$, H Mukhopadhyay ${ }^{39}$, A Mullavey ${ }^{44}$, J Munch ${ }^{47}$, P G Murray ${ }^{12}$, E Myers ${ }^{11}$, J Myers ${ }^{11}$, T Nash ${ }^{1}$, J Nelson ${ }^{12}$, G Newton ${ }^{12}$,

A Nishizawa $^{50}, \mathrm{~K}$ Numata $^{26}$, J O'Dell ${ }^{23}$, B O'Reilly ${ }^{19}$, R O'Shaughnessy ${ }^{38}$, E Ochsner $^{24}$, G H Ogin ${ }^{1}$, D J Ottaway ${ }^{47}$, R S Ottens ${ }^{6}$, H Overmier ${ }^{19}$,

B J Owen ${ }^{38}$, Y Pan ${ }^{24}$, C Pankow ${ }^{6}$, M A Papa ${ }^{3,9}$, V Parameshwaraiah ${ }^{11}$, P Patel ${ }^{1}$,

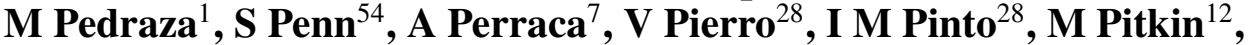

H J Pletsch ${ }^{2}$, M V Plissi ${ }^{12}$, F Postiglione ${ }^{36}$, M Principe ${ }^{28}$, R Prix ${ }^{2}$, L Prokhorov ${ }^{18}$,

O Punken ${ }^{2}$, V Quetschke ${ }^{6}$, F J Raab ${ }^{11}$, D S Rabeling ${ }^{44}$, H Radkins ${ }^{11}$, P Raffai ${ }^{35}$,

Z Raics ${ }^{15}$, N Rainer ${ }^{2}$, M Rakhmanov ${ }^{16}$, V Raymond ${ }^{49}, \mathbf{C} M$ Reed $^{11}$, T Reed ${ }^{55}$,

H Rehbein ${ }^{2}$, S Reid ${ }^{12}$, D H Reitze ${ }^{6}$, R Riesen ${ }^{19}$, K Riles ${ }^{27}$, B Rivera ${ }^{11}$,

P Roberts ${ }^{56}$, N A Robertson ${ }^{1,12}$, C Robinson ${ }^{34}$, E L Robinson ${ }^{9}$, S Roddy ${ }^{19}$,

C Röver ${ }^{2}$, J Rollins ${ }^{15}$, J D Romano ${ }^{16}$, J H Romie ${ }^{19}$, S Rowan ${ }^{12}$, A Rüdiger ${ }^{2}$,

P Russell ${ }^{1}$, K Ryan ${ }^{11}$, S Sakata ${ }^{50}$, L Sancho de la Jordana ${ }^{57}$, V Sandberg ${ }^{11}$,

V Sannibale $^{1}$, L Santamaría ${ }^{9}$, S Saraf ${ }^{58}$, P Sarin $^{14}$, B S Sathyaprakash ${ }^{34}$,

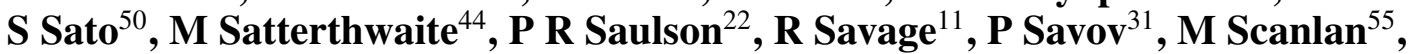

R Schilling ${ }^{2}$, R Schnabel ${ }^{2}$, R Schofield ${ }^{21}$, B Schulz ${ }^{2}$, B F Schutz ${ }^{9,34}$,

P Schwinberg ${ }^{11}$, J Scott ${ }^{12}$, S M Scott ${ }^{44}$, A C Searle ${ }^{1}$, B Sears ${ }^{1}$, F Seifert ${ }^{2}$,

D Sellers $^{19}$, A S Sengupta ${ }^{1}$, A Sergeev ${ }^{51}$, B Shapiro ${ }^{14}$, P Shawhan ${ }^{24}$,

D H Shoemaker ${ }^{14}$, A Sibley ${ }^{19}$, X Siemens ${ }^{3}$, D Sigg ${ }^{11}$, S Sinha ${ }^{4}$, A M Sintes ${ }^{57}$,

B J J Slagmolen ${ }^{44}$, J Slutsky ${ }^{5}$, J R Smith ${ }^{22}$, M R Smith ${ }^{1}$, N D Smith ${ }^{14}$,

K Somiya $^{31}$, B Sorazu ${ }^{12}$, A Stein ${ }^{14}$, L C Stein ${ }^{14}$, S Steplewski ${ }^{20}$, A Stochino ${ }^{1}$,

R Stone ${ }^{16}$, K A Strain ${ }^{12}$, S Strigin ${ }^{18}$, A Stroeer ${ }^{26}$, A L Stuver ${ }^{19}$,

T Z Summerscales ${ }^{56}$, K-X Sun ${ }^{4}$, M Sung ${ }^{5}$, P J Sutton ${ }^{34}$, G P Szokoly ${ }^{35}$,

D Talukder ${ }^{20}$, L Tang ${ }^{16}$, D B Tanner ${ }^{6}, \mathbf{S}$ P Tarabrin ${ }^{18}$, J R Taylor ${ }^{2}$, R Taylor ${ }^{1}$,

J Thacker $^{19}$, K A Thorne ${ }^{19}$, A Thüring ${ }^{8}$, K V Tokmakov ${ }^{12}$, C Torres $^{19}$,

C Torrie $^{1}$, G Traylor $^{19}$, M Trias ${ }^{57}$, D Ugolini ${ }^{59}$, J Ulmen ${ }^{4}$, K Urbanek ${ }^{4}$,

H Vahlbruch ${ }^{8}$, M Vallisneri ${ }^{31}$, C Van Den Broeck ${ }^{34}, M$ V van der Sluys ${ }^{49}$,

A A van Veggel ${ }^{12}, \mathbf{S}$ Vass $^{1}, \mathbf{R}$ Vaulin $^{3}$, A Vecchio $^{7}, \mathbf{J ~ V e i t c h}^{7}, \mathbf{P}$ Veitch $^{47}$,

C Veltkamp ${ }^{2}$, A Villar ${ }^{1}$, C Vorvick ${ }^{11}$, S P Vyachanin ${ }^{18}$, S J Waldman ${ }^{14}$,

L Wallace $^{1}$, R L Ward ${ }^{1}$, A Weidner ${ }^{2}$, M Weinert ${ }^{2}$, A J Weinstein ${ }^{1}$, R Weiss ${ }^{14}$,

L Wen ${ }^{13,31}$, S Wen ${ }^{5}$, K Wette ${ }^{44}$, J T Whelan ${ }^{9,60}$, S E Whitcomb ${ }^{1}$, B F Whiting ${ }^{6}$,

C Wilkinson $^{11}$, P A Willems ${ }^{1}$, H R Williams ${ }^{38}$, L Williams ${ }^{6}$, B Willke ${ }^{2,8}$,

I Wilmut ${ }^{23}$, L Winkelmann ${ }^{2}$, W Winkler ${ }^{2}$, C C Wipf ${ }^{14}$, A G Wiseman ${ }^{3}$,

G Woan ${ }^{12}, \mathbf{R}$ Wooley $^{19}, \mathbf{J}_{\text {Worden }}{ }^{11}, \mathbf{W} \mathbf{W u}^{6}$, I Yakushin $^{19}$, H Yamamoto ${ }^{1}$,

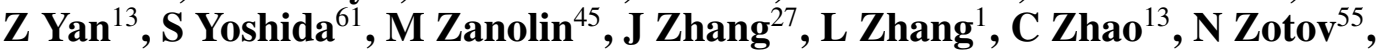

M E Zucker ${ }^{14}$, H zur Mühlen ${ }^{8}$ and J Zweizig ${ }^{1}$

\footnotetext{
${ }^{1}$ LIGO_California Institute of Technology, Pasadena, CA 91125, USA

2 Albert-Einstein-Institut, Max-Planck-Institut für Gravitationsphysik, D-30167 Hannover, Germany

${ }^{3}$ Department of Physics, University of Wisconsin-Milwaukee, Milwaukee, WI 53201, USA

${ }^{4}$ Ginzton, E.L. Laboratory, Stanford University, Stanford, CA 94305, USA

${ }^{5}$ Department of Physics and Astronomy, Louisiana State University, Baton Rouge, LA 70803, USA

${ }^{6}$ Department of Physics, University of Florida, Gainesville, FL 32611, USA
} 
${ }^{7}$ School of Physics and Astronomy, University of Birmingham, Birmingham, B15 2TT, UK

${ }^{8}$ Institut fur Gravitationsphysik, Leibniz Universität Hannover, D-30167 Hannover, Germany

${ }^{9}$ Albert-Einstein-Institut, Max-Planck-Institut für Gravitationsphysik, D-14476 Golm, Germany

${ }^{10}$ Department of Physics, Montana State University, Bozeman, MT 59717, USA

${ }^{11}$ LIGO-Hanford Observatory, Richland, WA 99352, USA

${ }^{12}$ Department of Physics and Astronomy, University of Glasgow, Glasgow, G12 8QQ, UK

${ }^{13}$ School of Physics, University of Western Australia, Crawley, WA 6009, Australia

${ }^{14}$ LIGO-Massachusetts Institute of Technology, Cambridge, MA 02139, USA

${ }^{15}$ Department of Physics, Columbia University, New York, NY 10027, USA

${ }^{16}$ Department of Physics, The University of Texas at Brownsville and Texas Southmost College, Brownsville, TX 78520, USA

${ }^{17}$ Department of Physics and Astronomy, San Jose State University, San Jose, CA 95192, USA

${ }^{18}$ Relativity Group, Moscow State University, Moscow, 119992, Russia

${ }^{19}$ LIGO-Livingston Observatory, Livingston, LA 70754, USA

${ }^{20}$ Department of Physics and Astronomy, Washington State University, Pullman, WA 99164, USA

${ }^{21}$ Department of Physics, University of Oregon, Eugene, OR 97403, USA

${ }^{22}$ Department of Physics, Syracuse University, Syracuse, NY 13244, USA

${ }^{23}$ Rutherford Appleton Laboratory, HSIC, Chilton, Didcot, Oxon OX11 0QX, UK

${ }^{24}$ Department of Physics, University of Maryland, College Park, MD 20742, USA

${ }^{25}$ Department of Physics, University of Massachusetts - Amherst, MA 01003, USA

${ }^{26}$ NASA/Goddard Space Flight Center, Greenbelt, MD 20771, USA

${ }^{27}$ Department of Physics, University of Michigan, Ann Arbor, MI 48109, USA

${ }^{28}$ Department of Engineering, University of Sannio at Benevento, I-82100 Benevento, Italy

${ }^{29}$ Department of Physics and Astronomy, The University of Mississippi, University, MS 38677, USA

${ }^{30}$ Research Scool of Physics and Engineering, Charles Sturt University, Wagga Wagga, NSW 2678, Australia

${ }^{31}$ Caltech-CaRT, Pasadena, CA 91125, USA

32 Department of Physics and Astronomy, Carleton College, Northfield, MN 55057, USA

${ }^{33}$ School of Physics, The University of Melbourne, Parkville VIC 3010, Australia

${ }^{34}$ School of Physics and Astronomy, Cardiff University, Cardiff CF24 3AA, UK

${ }^{35}$ Institute of Physics, Eötvös University, ELTE 1053 Budapest, Hungary

${ }^{36}$ Department of Physics, University of Salerno, 84084 Fisciano (Salerno), Italy

${ }^{37}$ Department of Physics and Astronomy, The University of Sheffield, Sheffield S10 2TN, UK

${ }^{38}$ Department of Astronomy and Astrophysics, The Pennsylvania State University, University Park, PA 16802, USA

${ }^{39}$ Inter-University Centre for Astronomy and Astrophysics, Pune - 411007, India

${ }^{40}$ Department of Physics, Southern University and A\&M College, Baton Rouge, LA 70813, USA

${ }^{41}$ California Institute of Technology, Pasadena, CA 91125, USA

${ }^{42}$ Department of Physics and Astronomy, University of Rochester, Rochester, NY 14627, USA

${ }^{43}$ Department of Physics, The University of Texas at Austin, Austin, TX 78712, USA

${ }^{44}$ Research School of Physics and Engineering, Australian National University, Canberra, 0200, Australia

${ }^{45}$ Department of Physics, Embry-Riddle Aeronautical University, Prescott, AZ 86301, USA

${ }^{46}$ Department of Physics, University of Minnesota, Minneapolis, MN 55455, USA

${ }^{47}$ School of Chemistry and Physics, University of Adelaide, Adelaide, SA 5005, Australia

${ }^{48}$ School of Physics and Astronomy, University of Southampton, Southampton, SO17 1BJ, UK

${ }^{49}$ Department of Astronomy and Astrophysics, Northwestern University, Evanston, IL 60208, USA

${ }^{50}$ National Astronomical Observatory of Japan, Tokyo 181-8588, Japan

${ }^{51}$ Institute of Applied Physics, Nizhny Novgorod, 603950, Russia

52 Department of Physics, University of Strathclyde, Glasgow, G1 1XQ, UK

${ }^{53}$ Department of Physics, Loyola University, New Orleans, LA 70118, USA

${ }^{54}$ Hobart and William Smith Colleges, Geneva, NY 14456, USA

${ }^{55}$ Department of Physics, Louisiana Tech University, Ruston, LA 71272, USA

${ }^{56}$ Department of Physics, Andrews University, Berrien Springs, MI 49104, USA

${ }^{57}$ Relativity and Gravitation Group, Universitat de les Illes Balears, E-07122 Palma de Mallorca, Spain

${ }^{58}$ Department of Physics and Astronomy, Sonoma State University, Rohnert Park, CA 94928, USA

${ }^{59}$ Department of Physics and Astronomy, Trinity University, San Antonio, TX 78212, USA

${ }^{60}$ Rochester Institute of Technology, Rochester, NY 14623, USA

${ }^{61}$ Southeastern Louisiana University, Hammond, LA 70402, USA

E-mail: peter.fritschel@ligo.org

Received 2 March 2009, in final form 1 May 2009

Published 30 June 2009

Online at stacks.iop.org/RoPP/72/076901 


\begin{abstract}
The goal of the Laser Interferometric Gravitational-Wave Observatory (LIGO) is to detect and study gravitational waves (GWs) of astrophysical origin. Direct detection of GWs holds the promise of testing general relativity in the strong-field regime, of providing a new probe of exotic objects such as black holes and neutron stars and of uncovering unanticipated new astrophysics. LIGO, a joint Caltech-MIT project supported by the National Science Foundation, operates three multi-kilometer interferometers at two widely separated sites in the United States. These detectors are the result of decades of worldwide technology development, design, construction and commissioning. They are now operating at their design sensitivity, and are sensitive to gravitational wave strains smaller than one part in $10^{21}$. With this unprecedented sensitivity, the data are being analyzed to detect or place limits on GWs from a variety of potential astrophysical sources.
\end{abstract}

(Some figures in this article are in colour only in the electronic version)

This article was invited by B Berger.

\title{
Contents
}

1. Introduction

2. Gravitational waves

3. LIGO and the worldwide detector network

4. Detector description

4.1. Interferometer configuration

4.2. Laser and optics

4.3. Suspensions and vibration isolation

4.4. Sensing and controls

4.5. Thermal effects

4.6. Interferometer response and calibration

4.7. Environmental monitors

5. Instrument performance

5.1. Strain noise spectra

5.2. Sensing noise sources
5.3. Seismic and thermal noise 12

5.4. Auxiliary degree-of-freedom noise 13

5.5. Actuation noise

5.6. Additional noise sources

5.7. Other performance figures-of-merit 14

6. Data analysis infrastructure 14

7. Astrophysical reach and search results 15

7.1. Compact binary coalescences 16

7.2. GW bursts 17

7.3. Continuous wave sources 19

7.4. Stochastic GW background 20

8. Future directions 22

Acknowledgments $\quad 22$

References $\quad 23$

\section{Introduction}

The prediction of gravitational waves (GWs), oscillations in the space-time metric that propagate at the speed of light, is one of the most profound differences between Einstein's general theory of relativity and the Newtonian theory of gravity that it replaced. GWs remained a theoretical prediction for more than 50 years until the first observational evidence for their existence came with the discovery and subsequent observations of the binary pulsar PSR $1913+16$, by Russell Hulse and Joseph Taylor. This is a system of two neutron stars (NSs) that orbit each other with a period of $7.75 \mathrm{~h}$. Precise timing of radio pulses emitted by one of the NSs, monitored now over several decades, shows that their orbital period is slowly decreasing at just the rate predicted for the general-relativistic emission of GWs [1]. Hulse and Taylor were awarded the Nobel Prize in Physics for this work in 1993.

In about 300 million years, the PSR $1913+16$ orbit will decrease to the point where the pair coalesces into a single compact object, a process that will produce directly detectable GWs. In the meantime, the direct detection of GWs will require similarly strong sources-extremely large masses moving with large accelerations in strong gravitational fields. The goal of LIGO, the Laser Interferometer
Gravitational-Wave Observatory [2], is just that: to detect and study GWs of astrophysical origin. Achieving this goal will mark the opening of a new window on the universe, with the promise of new physics and astrophysics. In physics, GW detection could provide information about strong-field gravitation, the untested domain of strongly curved space where Newtonian gravitation is no longer even a poor approximation. In astrophysics, the sources of GWs that LIGO might detect include binary NSs (like PSR $1913+16$ but much later in their evolution); binary systems where a black hole $(\mathrm{BH})$ replaces one or both of the NSs; a stellar core collapse which triggers a type II supernova; rapidly rotating, non-axisymmetric NSs; and possibly processes in the early universe that produce a stochastic background of GWs [3].

In the past few years the field has reached a milestone, with decades-old plans to build and operate large interferometric GW detectors now realized in several locations worldwide. This paper focuses on LIGO, which operates the most sensitive detectors yet built. We aim to describe the LIGO detectors and how they operate, explain how they have achieved their remarkable sensitivity and review how their data can be used to learn about a variety of astrophysical phenomena. 


\section{Gravitational waves}

The essence of general relativity is that mass and energy produce a curvature of four-dimensional space-time, and that matter moves in response to this curvature. The Einstein field equations prescribe the interaction between mass and space-time curvature, much as Maxwell's equations prescribe the relationship between electric charge and electromagnetic fields. Just as electromagnetic waves are time-dependent vacuum solutions to Maxwell's equations, GWs are timedependent vacuum solutions to the field equations. GWs are oscillating perturbations to a flat, or Minkowski, space-time metric, and can be thought of equivalently as an oscillating strain in space-time or as an oscillating tidal force between free test masses.

As with electromagnetic waves, GWs travel at the speed of light and are transverse in character, i.e. the strain oscillations occur in directions orthogonal to the direction in which the wave is propagating. Whereas electromagnetic waves are dipolar in nature, GWs are quadrupolar: the strain pattern contracts space along one transverse dimension, while expanding it along the orthogonal direction in the transverse plane (see figure 1). Gravitational radiation is produced by oscillating multipole moments of the mass distribution of a system. The principle of mass conservation rules out monopole radiation, and the principles of linear and angular momentum conservation rule out gravitational dipole radiation. Quadrupole radiation is the lowest allowed form and is thus usually the dominant form. In this case, the GW field strength is proportional to the second time derivative of the quadrupole moment of the source, and it falls off in amplitude inversely with distance from the source. The tensor character of gravity - the hypothetical graviton is a spin-2 particle-means that the transverse strain field comes in two orthogonal polarizations. These are commonly expressed in a linear polarization basis as the ' + ' polarization (depicted in figure 1) and the ' $x$ ' polarization, reflecting the fact that they are rotated $45^{\circ}$ relative to one another. An astrophysical GW will, in general, be a mixture of both polarizations.

GWs differ from electromagnetic waves in that they propagate essentially unperturbed through space, as they interact only very weakly with matter. Furthermore, GWs are intrinsically non-linear, because the wave energy density itself generates additional curvature of space-time. This phenomenon is only significant, however, very close to strong sources of waves, where the wave amplitude is relatively large. More usually, GWs distinguish themselves from electromagnetic waves by the fact that they are very weak. One cannot hope to detect any waves of terrestrial origin, whether naturally occurring or manmade; instead one must look for very massive compact astrophysical objects, moving at relativistic velocities. For example, strong sources of GWs that may exist in our galaxy or nearby galaxies are expected to produce wave strengths on Earth that do not exceed strain levels of one part in $10^{21}$. Finally, it is important to appreciate that GW detectors respond directly to GW amplitude rather than $\mathrm{GW}$ power; therefore the volume of space that is probed for potential sources increases as the cube of the strain sensitivity.

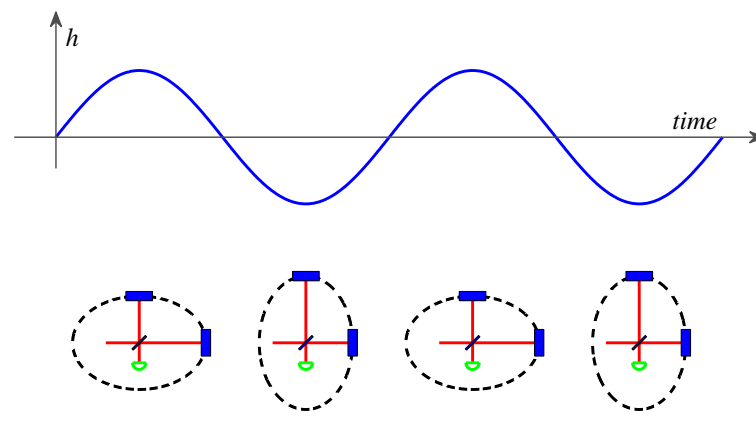

Figure 1. A GW traveling perpendicular to the plane of the diagram is characterized by a strain amplitude $h$. The wave distorts a ring of test particles into an ellipse, elongated in one direction in one

half-cycle of the wave, and elongated in the orthogonal direction in the next half-cycle. This oscillating distortion can be measured with a Michelson interferometer oriented as shown. The length oscillations modulate the phase shifts accrued by the light in each arm, which are in turn observed as light intensity modulations at the photodetector (green semi-circle). This depicts one of the linear polarization modes of the $\mathrm{GW}$.

\section{LIGO and the worldwide detector network}

As illustrated in figure 1, the oscillating quadrupolar strain pattern of a GW is well matched by a Michelson interferometer, which makes a very sensitive comparison of the lengths of its two orthogonal arms. LIGO utilizes three specialized Michelson interferometers, located at two sites (see figure 2): an observatory on the Hanford site in Washington houses two interferometers, the $4 \mathrm{~km}$-long $\mathrm{H} 1$ and $2 \mathrm{~km}$-long $\mathrm{H} 2$ detectors; and an observatory in Livingston Parish, Louisiana, houses the $4 \mathrm{~km}$-long L1 detector. Other than the shorter length of $\mathrm{H} 2$, the three interferometers are essentially identical. Multiple detectors at separated sites are crucial for rejecting instrumental and environmental artifacts in the data, by requiring coincident detections in the analysis. Also, because the antenna pattern of an interferometer is quite wide, source localization requires triangulation using three separated detectors.

The initial LIGO detectors were designed to be sensitive to GWs in the frequency band $40-7000 \mathrm{~Hz}$, and capable of detecting a GW strain amplitude as small as $10^{-21}$ [2]. With funding from the National Science Foundation, the LIGO sites and detectors were designed by scientists and engineers from the California Institute of Technology and the Massachusetts Institute of Technology, constructed in the late 1990s, and commissioned over the first 5 years of this decade. From November 2005 to September 2007, they operated at their design sensitivity in a continuous data-taking mode. The data from this science run, known as S5, are being analyzed for a variety of GW signals by a group of researchers known as the LIGO Scientific Collaboration [4]. At the most sensitive frequencies, the instrument root-mean-square $(\mathrm{rms})$ strain noise has reached an unprecedented level of $3 \times 10^{-22}$ in a $100 \mathrm{~Hz}$ band.

Although in principle LIGO can detect and study GWs by itself, the potential to do astrophysics can be quantitatively and qualitatively enhanced by operation in a more extensive network. For example, the direction of travel of the GWs and 

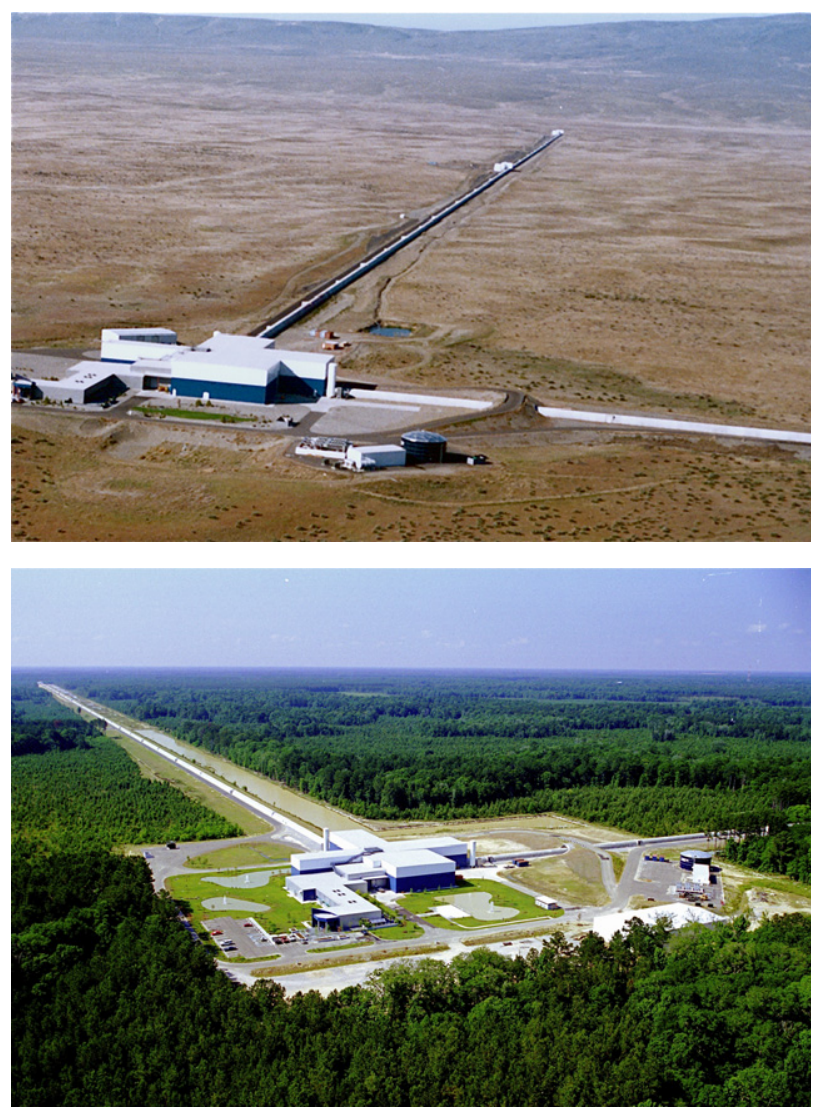

Figure 2. Aerial photograph of the LIGO observatories at Hanford, Washington (top) and Livingston, Louisiana (bottom). The lasers and optics are contained in the large corner buildings. From each corner building, evacuated beam tubes extend at right angles for $4 \mathrm{~km}$ in each direction (the full length of only one of the arms is seen in each photo); the tubes are covered by the arched, concrete enclosures seen here.

the complete polarization information carried by the waves can only be extracted by a network of detectors. Such a global network of GW observatories has been emerging over the past decade. In this period, the Japanese TAMA project built a $300 \mathrm{~m}$ interferometer outside Tokyo, Japan [5]; the GermanBritish GEO project built a $600 \mathrm{~m}$ interferometer near Hanover, Germany [6]; and the European Gravitational Observatory built the $3 \mathrm{~km}$-long interferometer Virgo near Pisa, Italy [7]. In addition, plans are underway to develop a large scale $\mathrm{GW}$ detector in Japan sometime during the next decade [8].

Early in its operation LIGO joined with the GEO project; for strong sources the shorter, less sensitive GEO 600 detector provides added confidence and directional and polarization information. In May 2007 the Virgo detector began joint observations with LIGO, with a strain sensitivity close to that of LIGO's $4 \mathrm{~km}$ interferometers at frequencies above $\sim 1 \mathrm{kHz}$. The LIGO Scientific Collaboration and the Virgo Collaboration negotiated an agreement that all data collected from that date are to be analyzed and published jointly.

\section{Detector description}

Figure 1 illustrates the basic concept of how a Michelson interferometer is used to measure a GW strain. The challenge is to make the instrument sufficiently sensitive: at the targeted strain sensitivity of $10^{-21}$, the resulting arm length change is only $\sim 10^{-18} \mathrm{~m}$, a thousand times smaller than the diameter of a proton. Meeting this challenge involves the use of special interferometry techniques, state-of-the-art optics, highly stable lasers and multiple layers of vibration isolation, all of which are described in the sections that follow. And of course a key feature of the detectors is simply their scale: the arms are made as long as practically possible to increase the signal due to a GW strain. See table 1 for a list of the main design parameters of the LIGO interferometers.

\subsection{Interferometer configuration}

The LIGO detectors are Michelson interferometers whose mirrors also serve as gravitational test masses. A passing $\mathrm{GW}$ will impress a phase modulation on the light in each arm of the Michelson, with a relative phase shift of $180^{\circ}$ between the arms. When the Michelson arm lengths are set such that the un-modulated light interferes destructively at the anti-symmetric (AS) port - the dark fringe condition-the phase modulated sideband light will interfere constructively, with an amplitude proportional to GW strain and the input power. With dark fringe operation, the full power incident on the beamsplitter is returned to the laser at the symmetric port. Only differential motion of the arms appears at the AS port; common mode signals are returned to the laser with the carrier light.

Two modifications to a basic Michelson, shown in figure 3, increase the carrier power in the arms and hence the GW sensitivity. First, each arm contains a resonant Fabry-Perot optical cavity made up of a partially transmitting input mirror and a high reflecting end mirror. The cavities cause the light to effectively bounce back and forth multiple times in the arms, increasing the carrier power and phase shift for a given strain amplitude. In the LIGO detectors the Fabry-Perot cavities multiply the signal by a factor of 100 for a $100 \mathrm{~Hz}$ GW. Second, a partially reflecting mirror is placed between the laser and beamsplitter to implement power recycling [9]. In this technique, an optical cavity is formed between the power recycling mirror and the Michelson symmetric port. By matching the transmission of the recycling mirror to the optical losses in the Michelson, and resonating this recycling cavity, the laser power stored in the interferometer can be significantly increased. In this configuration, known as a power recycled Fabry-Perot Michelson, the LIGO interferometers increase the power in the arms by a factor of $\approx 8000$ with respect to a simple Michelson.

\subsection{Laser and optics}

The laser source is a diode-pumped, Nd: YAG master oscillator and power amplifier system, and emits $10 \mathrm{~W}$ in a single frequency at $1064 \mathrm{~nm}$ [10]. The laser power and frequency are actively stabilized, and passively filtered with a transmissive ring cavity (pre-mode cleaner, PMC). The laser power stabilization is implemented by directing a sample of the beam to a photodetector, filtering its signal and feeding it back to the power amplifier; this servo stabilizes the relative power 
Table 1. Parameters of the LIGO interferometers. H1 and $\mathrm{H} 2$ refer to the interferometers at Hanford, Washington, and L1 is the interferometer at Livingston Parish, Louisiana.

\begin{tabular}{|c|c|c|c|}
\hline & $\mathrm{H} 1$ & L1 & $\mathrm{H} 2$ \\
\hline Laser type and wavelength & \multicolumn{3}{|c|}{$\mathrm{Nd}: \mathrm{YAG}, \lambda=1064 \mathrm{~nm}$} \\
\hline Arm cavity finesse & \multicolumn{3}{|c|}{220} \\
\hline Arm length (m) & 3995 & 3995 & 2009 \\
\hline Arm cavity storage time, $\tau_{s}(\mathrm{~ms})$ & 0.95 & 0.95 & 0.475 \\
\hline Input power at recycling mirror (W) & 4.5 & 4.5 & 2.0 \\
\hline Power Recycling gain & 60 & 45 & 70 \\
\hline Arm cavity stored power $(\mathrm{kW})$ & 20 & 15 & 10 \\
\hline Test mass size and mass & \multicolumn{3}{|c|}{$\varnothing 25 \mathrm{~cm} \times 10 \mathrm{~cm}, 10.7 \mathrm{~kg}$} \\
\hline $\begin{array}{l}\text { Beam radius }\left(1 / e^{2} \text { power) ITM/ETM }\right. \\
\text { Test mass pendulum frequency }(\mathrm{Hz})\end{array}$ & $3.6 \mathrm{~cm} / 4.5 \mathrm{~cm}$ & $\begin{array}{c}3.9 \mathrm{~cm} / 4.5 \mathrm{~cm} \\
0.76\end{array}$ & $3.3 \mathrm{~cm} / 3.5 \mathrm{~cm}$ \\
\hline
\end{tabular}

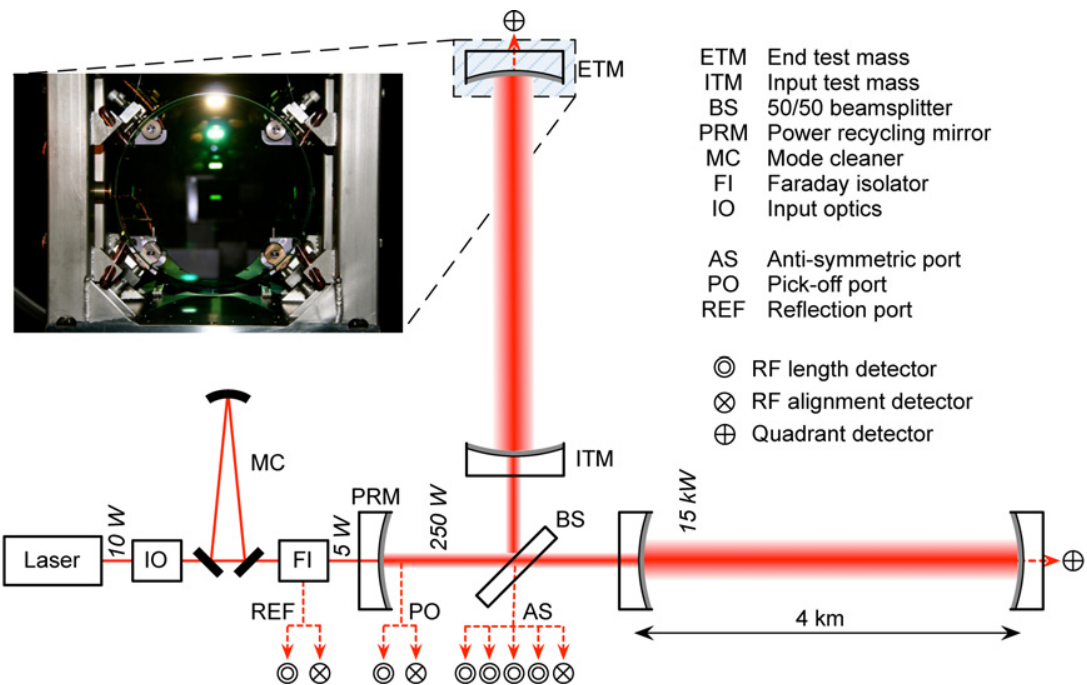

Figure 3. Optical and sensing configuration of the LIGO $4 \mathrm{~km}$ interferometers (the laser power numbers here are generic; specific power levels are given in table 1). The IO block includes laser frequency and amplitude stabilization, and electro-optic phase modulators. The power recycling cavity is formed between the PRM and the two ITMs, and contains the BS. The inset photo shows an input test mass mirror in its pendulum suspension. The near face has a highly reflective coating for the infrared laser light, but transmits visible light. Through it one can see mirror actuators arranged in a square pattern near the mirror perimeter.

fluctuations of the beam to $\sim 10^{-7} \mathrm{~Hz}^{-1 / 2}$ at $100 \mathrm{~Hz}[11]$. The laser frequency stabilization is done in multiple stages that are more fully described in later sections. The first, or pre-stabilization stage, uses the traditional technique of servo locking the laser frequency to an isolated reference cavity using the Pound-Drever-Hall (PDH) technique [12], in this case via feedback to frequency actuators on the master oscillator and to an electro-optic phase modulator. The servo bandwith is $500 \mathrm{kHz}$, and the pre-stabilization achieves a stability level of $\sim 10^{-2} \mathrm{~Hz} \mathrm{~Hz}^{-1 / 2}$ at $100 \mathrm{~Hz}$. The PMC transmits the pre-stabilized beam, filtering out both any light not in the fundamental Gaussian spatial mode and laser noise at frequencies above a few megahertz [13]. The PMC output beam is weakly phase-modulated with two radio-frequency (RF) sine waves, producing, to first-order, two pairs of sideband fields around the carrier field; these RF sideband fields are used in a heterodyne detection system described below.

After phase modulation, the beam passes into the LIGO vacuum system. All the main interferometer optical components and beam paths are enclosed in the ultra-high vacuum system $\left(10^{-8}-10^{-9}\right.$ Torr $)$ for acoustical isolation and to reduce phase fluctuations from light scattering off residual gas [14]. The long beam tubes are particularly noteworthy components of the LIGO vacuum system. These $1.2 \mathrm{~m}$ diameter, $4 \mathrm{~km}$ long stainless steel tubes were designed to have low-outgassing so that the required vacuum could be attained by pumping only from the ends of the tubes. This was achieved by special processing of the steel to remove hydrogen, followed by an in situ bakeout of the spiral-welded tubes, for approximately 20 days at $160^{\circ} \mathrm{C}$.

The in-vacuum beam first passes through the mode cleaner (MC), a $12 \mathrm{~m}$ long, vibrationally isolated transmissive ring cavity. The MC provides a stable, diffraction-limited beam with additional filtering of laser noise above several kilohertz [15], and it serves as an intermediate reference for frequency stabilization. The MC length and modulation frequencies are matched so that the main carrier field and the modulation sideband fields all pass through the MC. After the MC is a Faraday isolator and a reflective 3-mirror telescope that expands the beam and matches it to the arm cavity mode.

The interferometer optics, including the test masses, are fused-silica substrates with multilayer dielectric coatings, manufactured to have extremely low scatter and low 
absorption. The test mass substrates are polished so that the surface deviation from a spherical figure, over the central $80 \mathrm{~mm}$ diameter, is typically $5 \AA$ or smaller, and the surface microroughness is typically less than $2 \AA$ [16]. The mirror coatings are made using ion-beam sputtering, a technique known for producing ultralow-loss mirrors $[17,18]$. The absorption level in the coatings is generally a few parts-permillion (ppm) or less [19], and the total scattering loss from a mirror surface is estimated to be $60-70 \mathrm{ppm}$.

In addition to being a source of optical loss, scattered light can be a problematic noise source, if it is allowed to reflect or scatter from a vibrating surface (such as a vacuum system wall) and recombine with the main beam [20]. Since the vibrating, re-scattering surface may be moving by $\sim 10$ orders of magnitude more than the test masses, very small levels of scattered light can contaminate the output. To control this, various baffles are employed within the vacuum system to trap scattered light $[20,21]$. Each $4 \mathrm{~km}$ long beam tube contains approximately 200 baffles to trap light scattered at small angles from the test masses. These baffles are stainless steel truncated cones, with serrated inner edges, distributed so as to completely hide the beam tube from the line of sight of any arm cavity mirror. Additional baffles within the vacuum chambers prevent light outside the mirror apertures from hitting the vacuum chamber walls.

\subsection{Suspensions and vibration isolation}

Starting with the MC, each mirror in the beam line is suspended as a pendulum by a loop of steel wire. The pendulum provides $f^{-2}$ vibration isolation above its eigenfrequencies, allowing free movement of a test mass in the GW frequency band. Along the beam direction, a test mass pendulum isolates by a factor of nearly $2 \times 10^{4}$ at $100 \mathrm{~Hz}$. The position and orientation of a suspended optic is controlled by electromagnetic actuators: small magnets are bonded to the optic and coils are mounted to the suspension support structure, positioned to maximize the magnetic force and minimize ground noise coupling. The actuator assemblies also contain optical sensors that measure the position of the suspended optic with respect to its support structure. These signals are used to actively damp eigenmodes of the suspension.

The bulk of the vibration isolation in the $\mathrm{GW}$ band is provided by four-layer mass-spring isolation stacks, to which the pendulums are mounted. These stacks provide approximately $f^{-8}$ isolation above $\sim 10 \mathrm{~Hz}$ [22], giving an isolation factor of about $10^{8}$ at $100 \mathrm{~Hz}$. In addition, the L1 detector, subject to higher environmental ground motion than the Hanford detectors, employs seismic pre-isolators between the ground and the isolation stacks. These active isolators employ a collection of motion sensors, hydraulic actuators and servo controls; the pre-isolators actively suppress vibrations in the band $0.1-10 \mathrm{~Hz}$, by as much as a factor of 10 in the middle of the band [23].

\subsection{Sensing and controls}

The two Fabry-Perot arms and power recycling cavities are essential to achieving the LIGO sensitivity goal, but they require an active feedback system to maintain the interferometer at the proper operating point [24]. The round trip length of each cavity must be held to an integer multiple of the laser wavelength so that newly introduced carrier light interferes constructively with light from previous round trips. Under these conditions the light inside the cavities builds up and they are said to be on resonance. In addition to the three cavity lengths, the Michelson phase must be controlled to ensure that the AS port remains on the dark fringe.

The four lengths are sensed with a variation of the PDH reflection scheme [25]. In standard $\mathrm{PDH}$, an error signal is generated through heterodyne detection of the light reflected from a cavity. The RF phase modulation sidebands are directly reflected from the cavity input mirror and serve as a local oscillator to mix with the carrier field. The carrier experiences a phase shift in reflection, turning the RF phase modulation into RF amplitude modulation, linear in amplitude for small deviations from resonance. This concept is extended to the full interferometer as follows. At the operating point, the carrier light is resonant in the arm and recycling cavities and on a Michelson dark fringe. The RF sideband fields resonate differently. One pair of RF sidebands (from phase modulation at $62.5 \mathrm{MHz}$ ) is not resonant and simply reflects from the recycling mirror. The other pair ( $25 \mathrm{MHz}$ phase modulation) is resonant in the recycling cavity but not in the arm cavities. ${ }^{62}$ The Michelson mirrors are positioned to make one arm $30 \mathrm{~cm}$ longer than the other so that these RF sidebands are not on a Michelson dark fringe. By design this Michelson asymmetry is chosen so that most of the resonating RF sideband power is coupled to the AS port.

In this configuration, heterodyne error signals for the four length degrees-of-freedom are extracted from the three output ports shown in figure 3 (REF, PO and AS ports). The AS port is heterodyned at the resonating RF frequency and gives an error signal proportional to differential arm length changes, including those due to a GW. The PO port is a sample of the recycling cavity beam, and is detected at the resonating RF frequency to give error signals for the recycling cavity length and the Michelson phase (using both RF quadratures). The REF port is detected at the non-resonating RF frequency and gives a standard PDH signal proportional to deviations in the laser frequency relative to the average length of the two arms.

Feedback controls derived from these errors signals are applied to the two end mirrors to stabilize the differential arm length, to the beamsplitter to control the Michelson phase and to the recycling mirror to control the recycling cavity length. The feedback signals are applied directly to the mirrors through their coil-magnet actuators, with slow corrections for the differential arm length applied with longer range actuators that move the whole isolation stack.

The common arm length signal from the REF port detection is used in the final level of laser frequency stabilization [26] pictured schematically in figure 4 . The hierarchical frequency control starts with the reference cavity pre-stabilization mentioned in section 4.2. The prestabilization path includes an acousto-optic modulator (AOM)

62 These are approximate modulation frequencies for $\mathrm{H} 1$ and $\mathrm{L} 1$; those for $\mathrm{H} 2$ are about $10 \%$ higher. 


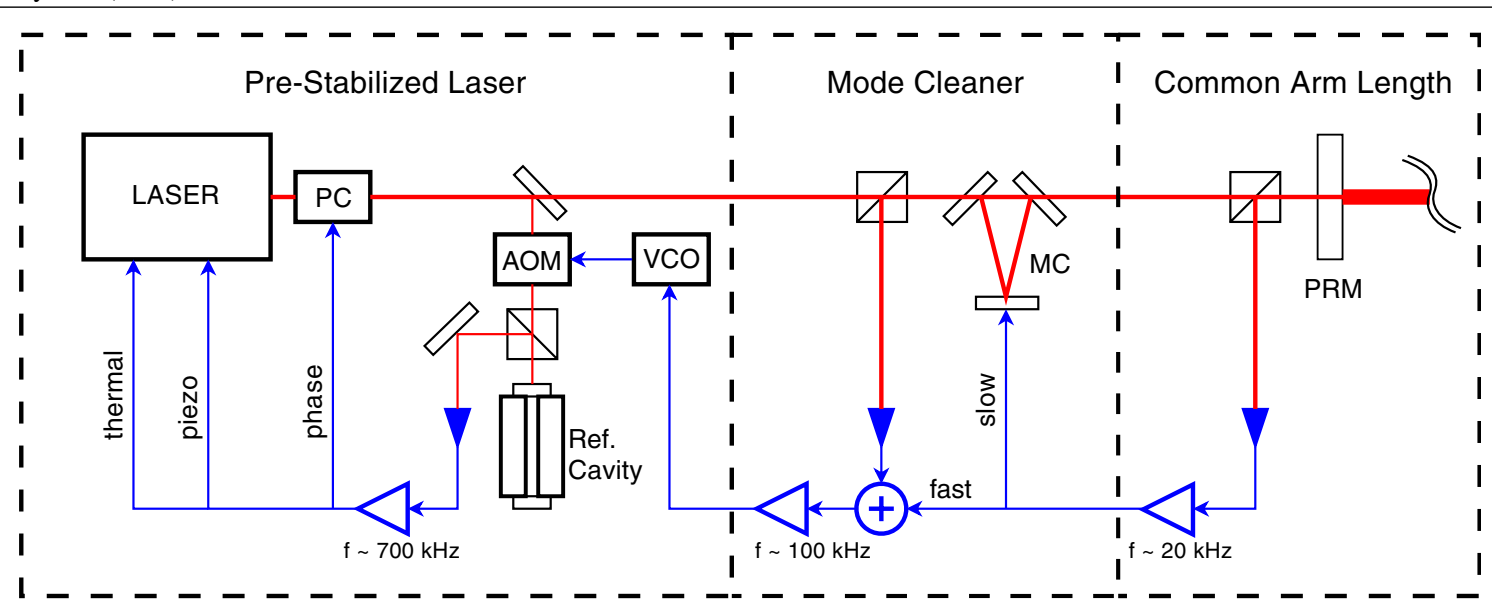

Figure 4. Schematic layout of the frequency stabilization servo. The laser is locked to a fixed-length reference cavity through an AOM. The AOM frequency is generated by a voltage controlled oscillator (VCO) driven by the MC servo, which is in turn driven by the common mode arm length signal from the REF port. The laser frequency is actuated by a combination of a pockels cell (PC), piezo-actuator and thermal control.

driven by a voltage-controlled oscillator, through which fast corrections to the pre-stabilized frequency can be made. The MC servo uses this correction path to stabilize the laser frequency to the MC length, with a servo bandwidth close to $100 \mathrm{kHz}$. The most stable frequency reference in the GW band is naturally the average length of the two arm cavities, therefore the common arm length error signal provides the final level of frequency correction. This is accomplished with feedback to the MC, directly to the MC length at low frequencies and to the error point of the MC servo at high frequencies, with an overall bandwidth of $20 \mathrm{kHz}$. The MC servo then impresses the corrections onto the laser frequency. The three cascaded frequency loops - the reference cavity pre-stabilization, the MC loop and the common arm length loop - together provide $160 \mathrm{~dB}$ of frequency noise reduction at $100 \mathrm{~Hz}$, and achieve a frequency stability of $5 \mu \mathrm{Hz}$ rms in a $100 \mathrm{~Hz}$ bandwidth.

The photodetectors are all located outside the vacuum system, mounted on optical tables. Telescopes inside the vacuum reduce the beam size by a factor of $\sim 10$, and the small beams exit the vacuum through high-quality windows. To reduce noise from scattered light and beam clipping, the optical tables are housed in acoustical enclosures, and the more critical tables are mounted on passive vibration isolators. Any back-scattered light along the AS port path is further mitigated with a Faraday isolator mounted in the vacuum system.

The total AS port power is typically $200-250 \mathrm{~mW}$, and is a mixture of RF sideband local oscillator power and carrier light resulting from spatially imperfect interference at the beamsplitter. The light is divided equally between four length photodetectors, keeping the power on each at a detectable level of 50-60 mW. The four length detector signals are summed and filtered, and the feedback control signal is applied differentially to the end test masses. This differential-arm servo loop has a unity-gain bandwidth of approximately $200 \mathrm{~Hz}$, suppressing fluctuations in the arm lengths to a residual level of $\sim 10^{-14} \mathrm{~m}$ rms. An additional servo is implemented on these AS port detectors to cancel signals in the RF phase orthogonal to the differential-arm channel; this servo injects RF current at each photodetector to suppress signals that would otherwise saturate the detectors. About $1 \%$ of the beam is directed to an alignment detector that controls the differential alignment of the ETMs.

Maximal power buildup in the interferometer also depends on maintaining stringent alignment levels. Sixteen alignment degrees-of-freedom-pitch and yaw for each of the six interferometer mirrors and the input beam direction-are controlled by a hierarchy of feedback loops. First, orientation motion at the pendulum and isolation stack eigenfrequencies is suppressed locally at each optic using optical lever angle sensors. Second, global alignment is established with four RF quadrant photodetectors at the three output ports as shown in figure 3. These RF alignment detectors measure wavefront misalignments between the carrier and sideband fields in a spatial version of PDH detection [27,28]. Together the four detectors provide five linearly independent combinations of the angular deviations from optimal global alignment [29]. These error signals feed a multiple-input multiple-output control scheme to maintain the alignment within $\sim 10^{-8} \mathrm{rad} \mathrm{rms}$ of the optimal point, using bandwidths between $\sim 0.5$ and $\sim 5 \mathrm{~Hz}$ depending on the channel. Finally, slower servos hold the beam centered on the optics. The beam positions are sensed at the arm ends using dc quadrant detectors that receive the weak beam transmitted through the ETMs, and at the corner by imaging the beam spot scattered from the beamsplitter face with a CCD camera.

The length and alignment feedback controls are all implemented digitally, with a real-time sampling rate of 16384 samples s$^{-1}$ for the length controls and 2048 samples s $^{-1}$ for the alignment controls. The digital control system provides the flexibility required to implement the multiple-input, multiple-output feedback controls described above. The digital controls also allow complex filter shapes to be easily realized, lend the ability to make dynamic changes in filtering and make it simple to blend sensor and control signals. As an example, optical gain changes are compensated to first order to keep the loop gains constant in time by making real-time feed-forward corrections to the digital gain based on cavity power levels.

The digital controls are also essential to implementing the interferometer lock acquisition algorithm. So far this 
section has described how the interferometer is maintained at the operating point. The other function of the control system is to acquire lock: to initially stabilize the relative optical positions to establish the resonance conditions and bring them within the linear regions of the error signals. Before lock the suspended optics are only damped within their suspension structures; ground motion and the equivalent effect of input-light frequency fluctuations cause the four (real or apparent) lengths to fluctuate by $0.1-1 \mu \mathrm{m}$ rms over time scales of $0.5-10 \mathrm{~s}$. The probability of all four degreesof-freedom simultaneously falling within the $\sim 1 \mathrm{~nm}$ linear region of the resonance points is thus extremely small and a controlled approach is required. The basic approach of the lock acquisition scheme, described in detail in [30], is to control the degrees-of-freedom in sequence: first the powerrecycled Michelson is controlled, then a resonance of one arm cavity is captured and finally a resonance of the other arm cavity is captured to achieve full power buildup. A key element of this scheme is the real-time, dynamic calculation of a sensor transformation matrix to form appropriate length error signals throughout the sequence. The interferometers are kept in lock typically for many hours at a time, until lock is lost due to environmental disturbances, instrument malfunction or operator command.

\subsection{Thermal effects}

At full power operation, a total of $20-60 \mathrm{~mW}$ of light is absorbed in the substrate and in the mirror surface of each ITM, depending on their specific absorption levels. Through the thermo-optic coefficient of fused silica, this creates a weak, though not insignificant thermal lens in the ITM substrates [31]. Thermo-elastic distortion of the test mass reflecting surface is not significant at these absorption levels. While the ITM thermal lens has little effect on the carrier mode, which is determined by the arm cavity radii of curvature, it does affect the RF sideband mode supported by the recycling cavity. This in turn affects the power buildup and mode shape of the RF sidebands in the recycling cavity, and consequently the sensitivity of the heterodyne detection signals [32,33]. Achieving maximum interferometer sensitivity thus depends critically on optimizing the thermal lens and thereby the mode shape, a condition which occurs at a specific level of absorption in each ITM (approximately $50 \mathrm{~mW}$ ). To achieve this optimum mode over the range of ITM absorption and stored power levels, each ITM thermal lens is actively controlled by directing additional heating beams, generated from $\mathrm{CO}_{2}$ lasers, onto each ITM [34]. The power and shape of the heating beams are controlled to maximize the interferometer optical gain and sensitivity. The shape can be selected to have either a Gaussian radial profile to provide more lensing, or an annular radial profile to compensate for excess lensing.

\subsection{Interferometer response and calibration}

The GW channel is the digital error point of the differential-arm servo loop. In principle, the GW channel could be derived from any point within this loop. The error point is chosen because the dynamic range of this signal is relatively small, since the large low-frequency fluctuations are suppressed by the feedback loop. To calibrate the error point in strain, the effect of the feedback loop is divided out, and the interferometer response to a differential arm strain is factored in [35]; this process can be done either in the frequency domain or directly in the time domain. The absolute length scale is established using the laser wavelength, by measuring the mirror drive signal required to move through an interference fringe. The calibration is tracked during operation with sine waves injected into the differential-arm loop. The uncertainty in the amplitude calibration is approximately $\pm 10 \%$. Timing of the GW channel is derived from the Global Positioning System; the absolute timing accuracy of each interferometer is better than $\pm 10 \mu \mathrm{s}$.

The response of the interferometer output as a function of GW frequency is calculated in detail in [36-38]. In the longwavelength approximation, where the wavelength of the GW is much longer than the size of the detector, the response $R$ of a Michelson-Fabry-Perot interferometer is approximated by a single-pole transfer function:

$$
R(f) \propto \frac{1}{1+\mathrm{i} f / f_{\mathrm{p}}},
$$

where the pole frequency is related to the arm cavity storage time by $f_{\mathrm{p}}=1 / 4 \pi \tau_{\mathrm{s}}$. Above the pole frequency $\left(f_{\mathrm{p}}=85 \mathrm{~Hz}\right.$ for the LIGO $4 \mathrm{~km}$ interferometers), the amplitude response drops off as $1 / f$. As discussed below, the measurement noise above the pole frequency has a white (flat) spectrum, and so the strain sensitivity decreases proportionally to frequency in this region. The single-pole approximation is quite accurate, differing from the exact response by less than a percent up to $\sim 1 \mathrm{kHz}$ [38].

In the long-wavelength approximation, the interferometer directional response is maximal for GWs propagating orthogonally to the plane of the interferometer arms and linearly polarized along the arms. Other angles of incidence or polarizations give a reduced response, as depicted by the antenna patterns shown in figure 5. A single detector has blind spots on the sky for linearly polarized GWs.

\subsection{Environmental monitors}

To complete a LIGO detector, the interferometers described above are supplemented with a set of sensors to monitor the local environment. Seismometers and accelerometers measure vibrations of the ground and various interferometer components; microphones monitor acoustic noise at critical locations; magnetometers monitor fields that could couple to the test masses or electronics; radio receivers monitor RF power around the modulation frequencies. These sensors are used to detect environmental disturbances that can couple to the GW channel.

\section{Instrument performance}

\subsection{Strain noise spectra}

During the commissioning period, as the interferometer sensitivity was improved, several short science runs were 

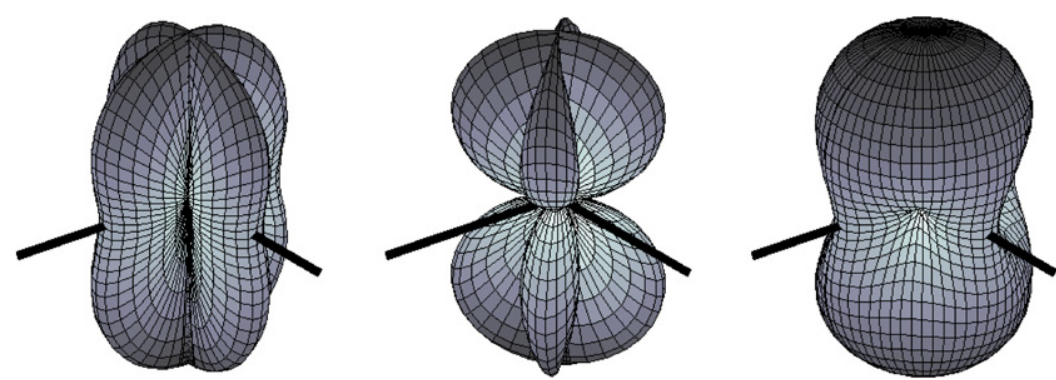

Figure 5. Antenna response pattern for a LIGO GW detector, in the long-wavelength approximation. The interferometer beamsplitter is located at the center of each pattern, and the thick black lines indicate the orientation of the interferometer arms. The distance from a point of the plot surface to the center of the pattern is a measure of the GW sensitivity in this direction. The pattern on the left is for + polarization, the middle pattern is for $\times$ polarization and the right-most one is for unpolarized waves.

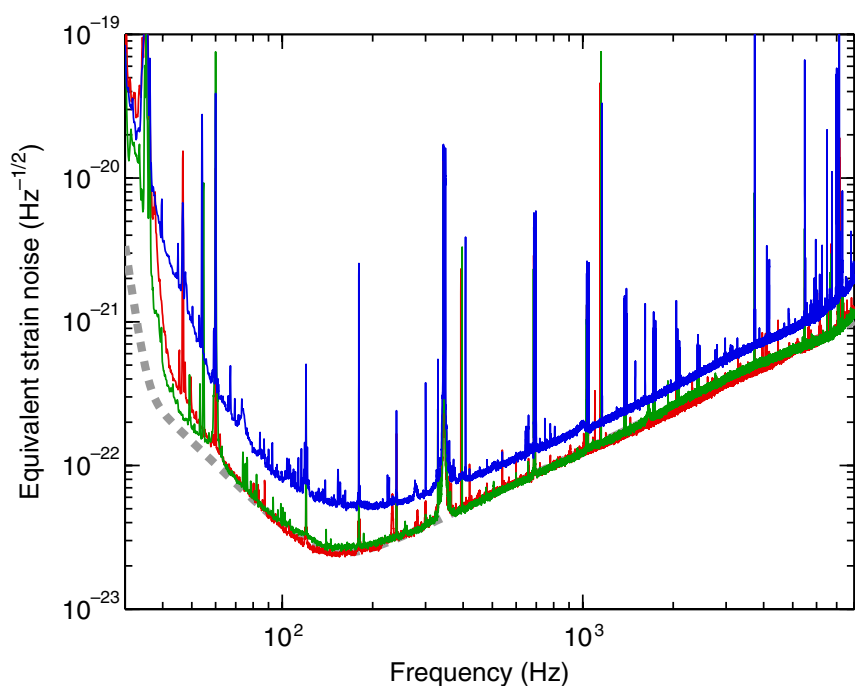

Figure 6. Strain sensitivities, expressed as amplitude spectral densities of detector noise converted to equivalent GW strain. The vertical axis denotes the rms strain noise in $1 \mathrm{~Hz}$ of bandwidth. Shown are typical high sensitivity spectra for each of the three interferometers (red: H1; blue: H2; green: L1), along with the design goal for the $4 \mathrm{~km}$ detectors (dashed gray).

carried out, culminating with the fifth science run (S5) at design sensitivity. The S5 run collected a full year of tripledetector coincident interferometer data during the period from November 2005 to September 2007. Since the interferometers detect GW strain amplitude, their performance is typically characterized by an amplitude spectral density of detector noise (the square root of the power spectrum), expressed in equivalent GW strain. Typical high-sensitivity strain noise spectra are shown in figure 6. Over the course of S5 the strain sensitivity of each interferometer was improved, by up to $40 \%$ compared with the beginning of the run through a series of incremental improvements to the instruments.

The primary noise sources contributing to the $\mathrm{H} 1$ strain noise spectrum are shown in figure 7 . Understanding and controlling these instrumental noise components has been the major technical challenge in the development of the detectors. The noise terms can be broadly divided into two classes: displacement noise and sensing noise. Displacement noises cause motions of the test masses or their mirrored surfaces. Sensing noises, on the other hand, are phenomena that limit the ability to measure those motions; they are present even in the absence of test mass motion. The strain noises shown in figure 6 consist of spectral lines superimposed on a continuous broadband noise spectrum. The majority of the lines are due to power lines $(60,120,180 \mathrm{~Hz}$, etc), 'violin mode' mechanical resonances $(350,700 \mathrm{~Hz}$, etc $)$ and calibration lines $(55,400$ and $1100 \mathrm{~Hz})$. These high $Q$ lines are easily excluded from analysis; the broadband noise dominates the instrument sensitivity.

\subsection{Sensing noise sources}

Sensing noises are shown in the lower panel of figure 7. By design, the dominant noise source above $100 \mathrm{~Hz}$ is shot noise, as determined by the Poisson statistics of photon detection. The ideal shot-noise limited strain noise density, $\tilde{h}(f)$, for this type of interferometer is [9]

$$
\tilde{h}(f)=\sqrt{\frac{\pi \hbar \lambda}{\eta P_{\mathrm{BS}} c}} \frac{\sqrt{1+\left(4 \pi f \tau_{\mathrm{s}}\right)^{2}}}{4 \pi \tau_{\mathrm{s}}},
$$

where $\lambda$ is the laser wavelength, $\hbar$ is the reduced Planck constant, $c$ is the speed of light, $\tau_{\mathrm{s}}$ is the arm cavity storage time, $f$ is the GW frequency, $P_{\mathrm{BS}}$ is the power incident on the beamsplitter and $\eta$ is the photodetector quantum efficiency. For the estimated effective power of $\eta P_{\mathrm{BS}}=0.9 \times 250 \mathrm{~W}$, the ideal shot-noise limit is $\tilde{h}=1.0 \times 10^{-23} \mathrm{~Hz}^{-1 / 2}$ at $100 \mathrm{~Hz}$. The shot-noise estimate in figure 7 is based on measured photocurrents in the AS port detectors and the measured interferometer response. The resulting estimate, $\tilde{h}(100 \mathrm{~Hz})=1.3 \times 10^{-23} \mathrm{~Hz}^{-1 / 2}$, is higher than the ideal limit due to several inefficiencies in the heterodyne detection process: imperfect interference at the beamsplitter increases the shot noise; imperfect modal overlap between the carrier and RF sideband fields decreases the signal and the fact that the AS port power is modulated at twice the RF phase modulation frequency leads to an increase in the time-averaged shot noise [39].

Many noise contributions are estimated using stimulusresponse tests, where a sine-wave or broadband noise is injected into an auxiliary channel to measure its coupling to the GW channel. This method is used for the laser frequency and amplitude noise estimates, the RF oscillator phase noise contribution and also for the angular control and auxiliary length noise terms described below. Although laser noise 

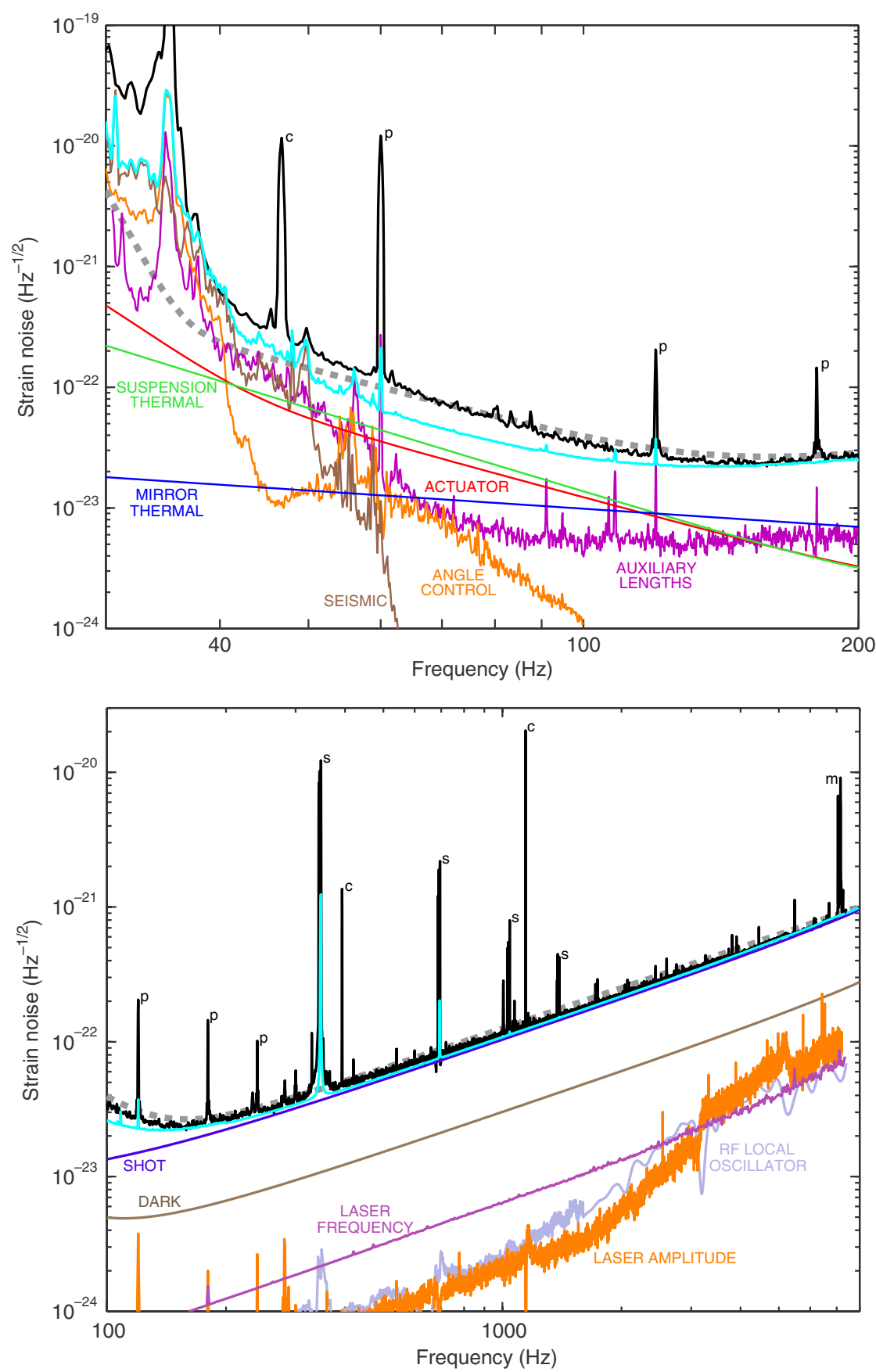

Figure 7. Primary known contributors to the $\mathrm{H} 1$ detector noise spectrum. The upper panel shows the displacement noise components, while the lower panel shows sensing noises (note the different frequency scales). In both panels, the black curve is the measured strain noise (same spectrum as in figure 6), the dashed gray curve is the design goal and the cyan curve is the root-square-sum of all known contributors (both sensing and displacement noises). The labeled component curves are described in the text. The known noise sources explain the observed noise very well at frequencies above $150 \mathrm{~Hz}$, and to within a factor of 2 in the $40-100 \mathrm{~Hz}$ band. Spectral peaks are identified as follows: c, calibration line; p, power line harmonic; s, suspension wire vibrational mode; m, mirror (test mass) vibrational mode.

is nominally common-mode, it couples to the GW channel through small, unavoidable differences in the arm cavity mirrors $[40,41]$. Frequency noise is expected to couple most strongly through a difference in the resonant reflectivity of the two arms. This causes carrier light to leak out the AS port, which interferes with frequency noise on the RF sidebands to create a noise signal. The stimulus-response measurements indicate that the coupling is due to a resonant reflectivity difference of about $0.5 \%$, arising from a loss difference of tens of ppm between the arms. Laser amplitude noise can couple through an offset from the carrier dark fringe. The measured coupling is linear, indicating an effective static offset of $\sim 1 \mathrm{pm}$, believed to be due to mode shape differences between the arms.

\subsection{Seismic and thermal noise}

Displacement noises are shown in the upper panel of figure 7. At the lowest frequencies the largest such noise is seismic 
noise-motions of the earth's surface driven by wind, ocean waves, human activity and low-level earthquakes - filtered by the isolation stacks and pendulums. The seismic contribution is estimated using accelerometers to measure the vibration at the isolation stack support points, and propagating this motion to the test masses using modeled transfer functions of the stack and pendulum. The seismic wall frequency, below which seismic noise dominates, is approximately $45 \mathrm{~Hz}$, a bit higher than the goal of $40 \mathrm{~Hz}$, as the actual environmental vibrations around these frequencies are $\sim 10$ times higher than what was estimated in the design.

Mechanical thermal noise is a more fundamental effect, arising from finite losses present in all mechanical systems, and is governed by the fluctuation-dissipation theorem $[42,43]$. It causes arm length noise through thermal excitation of the test mass pendulums (suspension thermal noise) [44], and thermal acoustic waves that perturb the test mass mirror surface (test mass thermal noise) [45]. Most of the thermal energy is concentrated at the resonant frequencies, which are designed (as much as possible) to be outside the detection band. Away from the resonances, the level of thermal motion is proportional to the mechanical dissipation associated with the motion. Designing the mirror and its pendulum to have very low mechanical dissipation reduces the detection-band thermal noise. It is difficult, however, to accurately and unambiguously establish the level of broadband thermal noise in situ; instead, the thermal noise curves in figure 7 are calculated from models of the suspension and test masses, with mechanical loss parameters taken from independent characterizations of the materials.

For the pendulum mode, the mechanical dissipation occurs near the ends of the suspension wire, where the wire flexes. Since the elastic energy in the flexing regions depends on the wire radius to the fourth power, it helps to make the wire as thin as possible to limit thermal noise. The pendulums are thus made with steel wire for its strength; with a diameter of $300 \mu \mathrm{m}$ the wires are loaded to $30 \%$ of their breaking stress. The thermal noise in the pendulum mode of the test masses is estimated assuming a frequency-independent mechanical loss angle in the suspension wire of $3 \times 10^{-4}$ [46]. This is a relatively small loss for a metal wire [47].

Thermal noise of the test mass surface is associated with mechanical damping within the test mass. The fusedsilica test mass substrate material has very low mechanical loss, of order $10^{-7}$ or smaller [48]. On the other hand, the thin-film, dielectric coatings that provide the required optical reflectivity - alternating layers of silicon dioxide and tantalum pentoxide - have relatively high mechanical loss. Even though the coatings are only a few micrometers thick, they are the dominant source of the relevant mechanical loss, due to their level of dissipation and the fact that it is concentrated on the test mass face probed by the laser beam [43]. The test mass thermal noise estimate is calculated by modeling the coatings as having a frequency-independent mechanical dissipation of $4 \times 10^{-4}[45]$.

\subsection{Auxiliary degree-of-freedom noise}

The auxiliary length noise term refers to noise in the Michelson and power recycling cavity servo loops which couple to the $\mathrm{GW}$ channel. The former couples directly to the GW channel while the latter couples in a manner similar to frequency noise. Above $\sim 50 \mathrm{~Hz}$ the sensing noise in these loops is dominated by shot noise; since loop bandwidths of $\sim 100 \mathrm{~Hz}$ are needed to adequately stabilize these degrees-of-freedom, shot noise is effectively added onto their motion. Their noise infiltration to the GW channel, however, is mitigated by appropriately filtering and scaling their digital control signals and adding them to the differential-arm control signal as a type of feedforward noise suppression [24]. These correction paths reduce the coupling to the GW channel by $10-40 \mathrm{~dB}$.

We illustrate this more concretely with the Michelson loop. The shot-noise-limited sensitivity for the Michelson is $\sim 10^{-16} \mathrm{~m} \mathrm{~Hz}^{-1 / 2}$. Around $100 \mathrm{~Hz}$, the Michelson servo impresses this sensing noise onto the Michelson degree-offreedom (specifically, onto the beamsplitter). Displacement noise in the Michelson couples to displacement noise in the GW channel by a factor of $\pi /(\sqrt{2} F)=1 / 100$, where $F$ is the arm cavity finesse. The Michelson sensing noise would thus produce $\sim 10^{-18} \mathrm{~m} \mathrm{~Hz}^{-1 / 2}$ of $\mathrm{GW}$ channel noise around $100 \mathrm{~Hz}$, if uncorrected. The digital correction path subtracts the Michelson noise from the GW channel with an efficiency of $95 \%$ or more. This brings the Michelson noise component down to $\sim 10^{-20} \mathrm{~m} \mathrm{~Hz}^{-1 / 2}$ in the GW channel, 5-10 times below the GW channel noise floor.

Angular control noise arises from noise in the alignment sensors (both optical levers and wavefront sensors), propagating to the test masses through the alignment control servos. Though these feedback signals affect primarily the test mass orientation, there is always some coupling to the GW degree-of-freedom because the laser beam is not perfectly aligned to the center-of-rotation of the test mass surface [49]. Angular control noise is minimized by a combination of filtering and parameter tuning. Angle control bandwidths are $10 \mathrm{~Hz}$ or less and strong low-pass filtering is applied in the GW band. In addition, the angular coupling to the GW channel is minimized by tuning the center-of-rotation, using the four actuators on each optic, down to typical residual coupling levels of $10^{-3}-10^{-4} \mathrm{~m} \mathrm{rad}^{-1}$.

\subsection{Actuation noise}

The actuator noise term includes the electronics that produce the coil currents keeping the interferometer locked and aligned, starting with the digital-to-analog converters (DACs). The actuation electronics chain has extremely demanding dynamic range requirements. At low frequencies, control currents of $\sim 10 \mathrm{~mA}$ are required to provide $\sim 5 \mu \mathrm{m}$ of position control, and tens of milliamperes are required to provide $\sim 0.5 \mathrm{mrad}$ of alignment bias. Yet the current noise through the coils must be kept below a couple of $\mathrm{pA} / \sqrt{\mathrm{Hz}}$ above $40 \mathrm{~Hz}$. The relatively limited dynamic range of the DACs is managed with a combination of digital and analog filtering: the higher frequency components of the control signals are digitally emphasized before being sent to the DACs, and 
then de-emphasized following the DACs with complementary analog filters. The dominant coil current noise comes instead from the circuits that provide the alignment bias currents, followed closely by the circuits that provide the length feedback currents.

\subsection{Additional noise sources}

In the $50-100 \mathrm{~Hz}$ band, the known noise sources typically do not fully explain the measured noise. Additional noise mechanisms have been identified, though not quantitatively established. Two potentially significant candidates are nonlinear conversion of low-frequency actuator coil currents to broadband noise (upconversion) and electric charge buildup on the test masses. A variety of experiments have shown that the upconversion occurs in the magnets (neodymium iron boron) of the coil-magnet actuators, and produces a broadband force noise, with a $f^{-2}$ spectral slope; this is the phenomenon known as Barkhausen noise [50]. The non-linearity is small but not negligible given the dynamic range involved: $0.1 \mathrm{mN}$ of low-frequency (below a few hertz) actuator force upconverts of order $10^{-11} \mathrm{~N}$ rms of force noise in the $40-80 \mathrm{~Hz}$ octave. This noise mechanism is significant primarily below $80 \mathrm{~Hz}$, and varies in amplitude with the level of ground motion at the observatories.

Regarding electric charge, mechanical contact of a test mass with its nearby limit-stops, as happens during a large earthquake, can build up charge between the two objects. Such charge distributions are not stationary; they tend to redistribute on the surface to reduce local charge density. This produces a fluctuating force on the test mass, with an expected $f^{-1}$ spectral slope. Although the level at which this mechanism occurs in the interferometers is not well known, evidence for its potential significance comes from a fortuitous event with L1. Following a vacuum vent and pump-out cycle partway through the S5 science run, the strain noise in the $50-100 \mathrm{~Hz}$ band went down by about $20 \%$; this was attributed to charge reduction on one of the test masses.

In addition to these broadband noises, there are a variety of periodic or quasi-periodic processes that produce lines or narrow features in the spectrum. The largest of these spectral peaks are identified in figure 7. The groups of lines around $350 \mathrm{~Hz}, 700 \mathrm{~Hz}$, etc are vibrational modes of the wires that suspend the test masses, thermally excited with $k T$ of energy in each mode. The power line harmonics, at $60 \mathrm{~Hz}, 120 \mathrm{~Hz}$, $180 \mathrm{~Hz}$, etc infiltrate the interferometer in a variety of ways. The $60 \mathrm{~Hz}$ line, for example, is primarily due to the power line's magnetic field coupling directly to the test mass magnets. As all these lines are narrow and fairly stable in frequency, they occupy only a small fraction of the instrument spectral bandwidth.

\subsection{Other performance figures-of-merit}

While figures 6 and 7 show high-sensitivity strain noise spectra, the interferometers exhibit both long- and short-term variation in sensitivity due to improvements made to the detectors, seasonal and daily variations in the environment, and the like. One indicator of the sensitivity variation over the S5 science

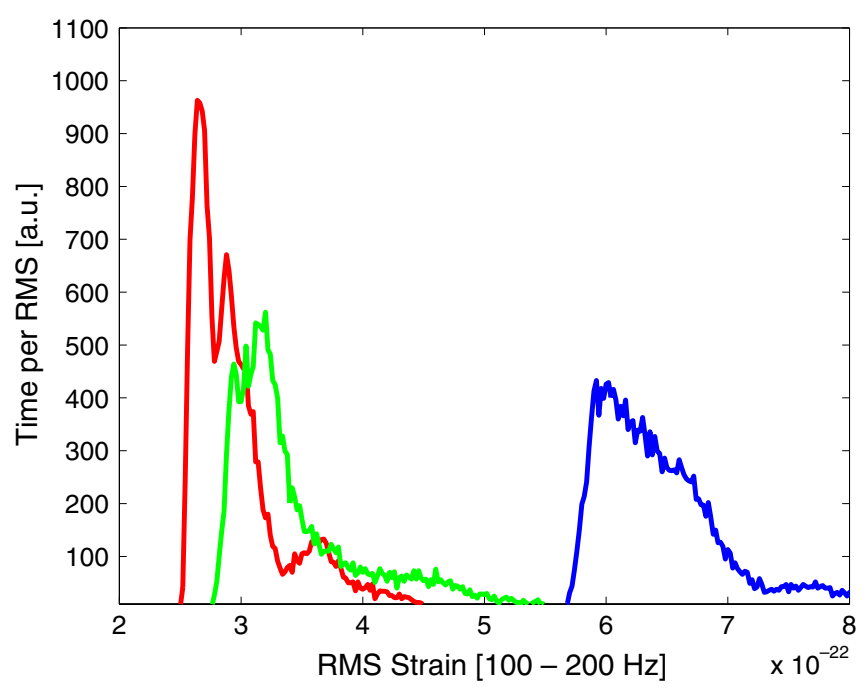

Figure 8. Histograms of the RMS strain noise in the band 100-200 Hz, computed from the S5 data for each of the LIGO interferometers (red: H1; green: L1; blue: H2). Each RMS strain value is calculated using $30 \mathrm{~min}$ of data. Much of the higher RMS portions of each distribution date from the first $\sim 100$ days of the run, around which time sensitivity improvements were made to all interferometers. Typical RMS variations over daily and weekly time scales are $\pm 5 \%$ about the mean. With the half arm length of $\mathrm{H} 2$, its RMS strain noise in this band is expected to be about two times higher than that of $\mathrm{H} 1$ and $\mathrm{L} 1$.

run is shown in figure 8: histograms of the rms strain noise in the frequency band of 100-200 Hz.

To get a sense of shorter term variations in the noise, figure 9 shows the distribution of strain noise amplitudes at three representative frequencies where the noise is dominated by random processes. For stationary, Gaussian noise the amplitudes would follow a Rayleigh distribution, and deviations from that indicate non-Gaussian fluctuations. As figure 9 suggests, the lower frequency end of the measurement band shows a higher level of non-Gaussian noise than the higher frequencies. Some of this non-Gaussianity is due to known couplings to a fluctuating environment; much of it, however, is due to glitches-any short duration noise transient-from unknown mechanisms. Additional characterizations of the glitch behavior of the detectors can be found in [51].

Another important statistical figure-of-merit is the interferometer duty cycle, the fraction of time that detectors are operating and taking science data. Over the S5 period, the individual interferometer duty cycles were 78\%,79\% and $67 \%$ for $\mathrm{H} 1, \mathrm{H} 2$ and $\mathrm{L} 1$, respectively; for double coincidence between $\mathrm{L} 1$ and $\mathrm{H} 1$ or $\mathrm{H} 2$ the duty cycle was $60 \%$; and for triple coincidence of all three interferometers the duty cycle was $54 \%$. These figures include scheduled maintenance and instrument tuning periods, as well as unintended losses of operation.

\section{Data analysis infrastructure}

While the LIGO interferometers provide extremely sensitive measurements of the strain at two distant locations, the 


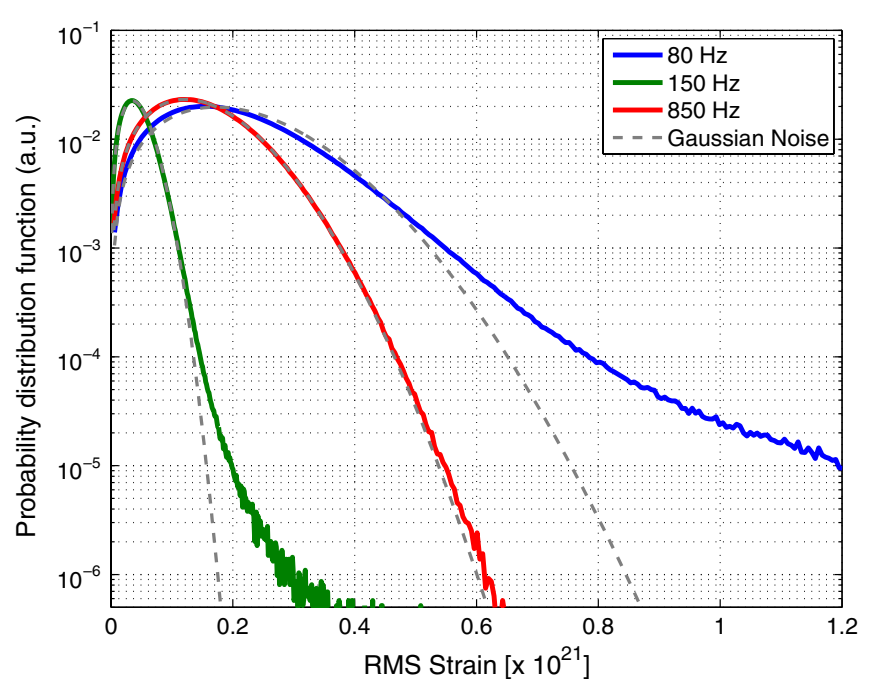

Figure 9. Distribution of strain noise amplitude for three representative frequencies within the measurement band (data shown for the $\mathrm{H} 1$ detector). Each curve is a histogram of the spectral amplitude at the specified frequency over the second half of the S5 data run. Each spectral amplitude value is taken from the Fourier transform of $1 \mathrm{~s}$ of strain data; the equivalent noise bandwidth for each curve is $1.5 \mathrm{~Hz}$. For comparison, the dashed gray lines are Rayleigh distributions, which the measured histograms would follow if they exhibited stationary, Gaussian noise. The high frequency curve is close to a Rayleigh distribution, since the noise there is dominated by shot noise. The lower frequency curves, on the other hand, exhibit non-Gaussian fluctuations.

instruments constitute only one half of the 'GravitationalWave Observatory' in LIGO. The other half is the computing infrastructure and data analysis algorithms required to pull out GW signals from the noise. Potential sources and the methods used to search for them are discussed in the next section. First, we discuss some features of the LIGO data and their analysis that are common to all searches.

The raw instrument data are collected and archived for offline analysis. For each detector, approximately 50 channels are recorded at a sample rate of $16384 \mathrm{~Hz}, 550$ channels at reduced rates of $256-4096 \mathrm{~Hz}$ and 6000 digital monitors at $16 \mathrm{~Hz}$. The aggregate rate of archived data is about $5 \mathrm{MB} \mathrm{s}^{-1}$ for each interferometer. Computer clusters at each site also produce reduced data sets containing only the most important channels for analysis.

The detector outputs are pre-filtered with a series of data quality checks to identify appropriate time periods to analyze. The most significant data quality (DQ) flag, 'science mode', ensures the detectors are in their optimum run-time configuration; it is set by the on-site scientists and operators. Follow-up DQ flags are set for impending lock loss, hardware injections, site disturbances and data corruptions. DQ flags are also used to mark times when the instrument is outside its nominal operating range, for instance when a sensor or actuator is saturating, or environmental conditions are unusually high. Depending on the specific search algorithm, the DQ flags introduce an effective dead-time of $1-10 \%$ of the total science mode data.

Injections of simulated GW signals are performed to test the functionality of all the search algorithms and also to measure detection efficiencies. These injections are done both in software, where the waveforms are added to the archived data stream, and directly in hardware, where they are added to the feedback control signal in the differential-arm servo. In general, the injected waveforms simulate the actual signals being searched for, with representative waveforms used to test searches for unknown signals.

As described in the section on instrument performance, the local environment and the myriad interferometer degreesof-freedom can all couple to the GW channel, potentially creating artifacts that must be distinguished from actual signals. Instrument-based vetoes are developed and used to reject such artifacts [51]. The vetoes are tested using hardware injections to ensure their safety for GW detections. The efficacy of these vetoes depends on the search type.

\section{Astrophysical reach and search results}

LIGO was designed so that its data could be searched for GWs from many different sources. The sources can be broadly characterized as either transient or continuous in nature, and for each type, the analysis techniques depend on whether the gravitational waveforms can be accurately modeled or whether only less specific spectral characterizations are possible. We therefore organize the searches into four categories according to source type and analysis technique.

(i) Transient, modeled waveforms: the compact binary coalescence search. The name follows from the fact that the best understood transient sources are the final stages of binary inspirals [52], where each component of the binary may be a NS or a BH. For these sources the waveform can be calculated with good precision, and matched-filter analysis can be used.

(ii) Transient, unmodeled waveforms: the gravitational-wave bursts search. Transient systems, such as core-collapse supernovae [53], BH mergers and NS quakes, may produce $\mathrm{GW}$ bursts that can only be modeled imperfectly, if at all, and more general analysis techniques are needed.

(iii) Continuous, narrow-band waveforms: the continuous wave sources search. An example of a continuous source of GWs with a well-modeled waveform is a spinning NS (e.g. a pulsar) that is not perfectly symmetric about its rotation axis [54].

(iv) Continuous, broadband waveforms: the stochastic gravitational-wave background search. Processes operating in the early universe, for example, could have produced a background of GWs that is continuous but stochastic in character [55].

In the following sections we review the astrophysical results that have been generated in each of these search categories using LIGO data; [56] contains links to all the LIGO observational publications. To date, no GW signal detections have been made, so these results are all upper limits on various GW sources. In those cases where the S5 analysis is not yet complete, we present the most recent published results and also discuss the expected sensitivity, or astrophysical reach, of the search based on the S5 detector performance. 


\subsection{Compact binary coalescences}

Binary coalescences are unique laboratories for testing general relativity in the strong-field regime [57]. GWs from such systems will provide unambiguous evidence for the existence of BHs and powerful tests of their properties as predicted by general relativity $[58,59]$. Multiple observations will yield valuable information about the population of such systems in the universe, up to distances of hundreds of megaparsecs (Mpc, 1 parsec $=3.3$ light years). Coalescences involving NSs will provide information about the nuclear equation of state in these extreme conditions. Such systems are considered likely progenitors of short-duration gamma-ray bursts (GRBs) [60].

Post-Newtonian approximations to general relativity accurately model a binary system of compact objects whose orbit is adiabatically tightening due to GW emission [61]. Several examples of such binary systems exist with merger times less than the age of the universe, most notably the binary pulsar system PSR $1913+16$ described previously. After an extended inspiral phase, the system becomes dynamically unstable when the separation decreases below an innermost stable circular orbit (approximately $25 \mathrm{~km}$ for two solarmass NSs) and the objects plunge and form a single $\mathrm{BH}$ in the merger phase. The resulting distorted $\mathrm{BH}$ relaxes to a stationary Kerr state via the strongly damped sinusoidal oscillations of the quasi-normal modes in the ringdown phase. The smoothly evolving inspiral and ringdown $\mathrm{GW}$ waveforms can be approximated analytically, while the extreme dynamics of the merger phase require numeric solutions to determine the GW waveform [62]. Collectively, the inspiral, merger and ringdown of a binary system is termed a compact binary coalescence $(\mathrm{CBC})$.

The waveform for a compact binary inspiral is a chirp: a sinusoid increasing in frequency and amplitude until the end of the inspiral phase. The inspiral phase of a binary neutron star (BNS, with each mass assumed to be $1.4 M_{\odot}$ ) will complete nearly 2000 orbits in the LIGO band over tens of seconds before merger, and emit a maximum GW frequency of about $1500 \mathrm{~Hz}$. Higher mass inspirals terminate at proportionally lower GW frequencies. For non-spinning objects, the inspiral waveform is uniquely determined by the two component masses $m_{1}$ and $m_{2}$ of the system [63]. No analytic waveforms exist for the merger phase; calculating these waveforms is one of the primary goals of numerical relativity $[64,65]$. The ringdown phase is described by an exponentially damped sinusoid, determined by the quasi-normal mode frequency and the quality factor of the final $\mathrm{BH}$ [66].

7.1.1. Analysis method. Since the inspiral and ringdown waveforms for a given mass pair $\left(m_{1}, m_{2}\right)$ are accurately known, searches for them are performed using optimal matched filtering employing a bank of templates covering the desired $\left(m_{1}, m_{2}\right)$ parameter space. An optimized algorithm generates the template bank, minimizing the number of templates while allowing a maximum signal-to-noise ratio (SNR) loss of 3\% [67-69]. In practice approximately 7000 templates are used to cover total masses between $2 M_{\odot}$ and $35 M_{\odot}$.
The matched filtering process generates a trigger when the SNR of the filter output exceeds a threshold. The threshold is set by balancing two factors: it must be low enough so that a good estimation can be made of the background due to detector noise, and it must be high enough to keep the number of triggers manageable. Associated with each trigger is a specific template, or mass pair, and a coalescence time which maximize the SNR for that signal event [70].

Triggers are first generated independently for each detector. The number of false triggers created by detector noise is then greatly reduced by finding the set of coincident triggers - those that correspond to similar template masses and coalescence times, within appropriate windows, between at least two LIGO detectors. Coincident triggers are subject to additional consistency checks, such as the $\chi^{2}$ [71] and $r^{2}[72]$ tests.

Typically many thousands of coincident triggers per month remain at the end of the pipeline. These surviving triggers are compared with the background from accidental coincidences of triggers due to detector noise. Time shift trials are used to estimate the background: the analysis is repeated with the triggers from different detectors shifted in time relative to each other by an amount large compared with the coincidence window. A hundred such trials are typically made. For each region of mass parameter space, the time shift trials establish a false alarm rate as a function of SNR. In-time coincident triggers with the smallest false alarm rate are potential detection candidates [73].

A large number of software injections is made to tune the analysis pipeline and evaluate its detection efficiency. The injected waveforms cover the largest practical range of parameter space possible (component masses, spins, orientations, sky locations and distances). The resulting detection efficiency is combined with simple models of the astrophysical source distribution to arrive at an estimated cumulative luminosity to which the search is sensitive. These models $[74,75]$ predict that the rate of CBCs should be proportional to the stellar birth rate in nearby spiral galaxies. This birth rate can be estimated from a galaxy's blue luminosity ${ }^{63}$, so we express the cumulative luminosity in units of $L_{10}$, where $L_{10}$ is $10^{10}$ times the blue solar luminosity (the Milky Way contains $\sim 1.7 L_{10}$ )

7.1.2. Analysis results. To date, the detection candidates resulting from the analysis pipeline are consistent with the estimated background and thus are likely accidental coincidences. In the absence of detection, mass-dependent upper limits are set on the rate of CBCs in the local universe. These rate limits are expressed per unit $L_{10}$.

An inspiral search with total masses between $2 M_{\odot}$ and $35 M_{\odot}$ has been completed using the first calendar year of S5 data [73]. Figure 10 shows the resulting rate upper limit for low-mass binary coalescences as a function of the total mass (left), and as a function of the mass of a BH in a BH-NS system with a NS mass of $1.35 M_{\odot}$ (right). The same analysis set a BNS coalescence rate upper limit of $3.8 \times 10^{-2} \mathrm{yr}^{-1} L_{10}^{-1}$.

${ }^{63}$ Blue luminosity is short for B-band luminosity, signifying one of a standard set of optical filters used in measuring the luminosity of galaxies. 

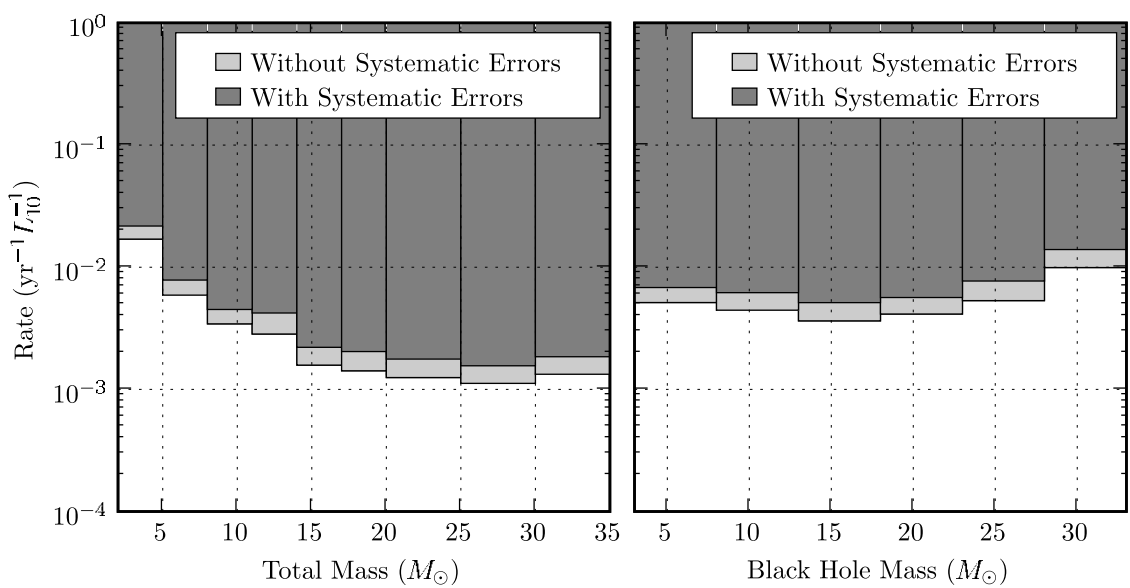

Figure 10. S5 year 1 upper limits on the binary coalescence rate per year and per L10 as a function of total mass of the binary system assuming a uniform distribution in the mass ratio (left) and as a function of the mass of a BH in a BH-NS system with a NS mass of $1.35 M_{\odot}$ (right). The darker area shows the excluded region when accounting for marginalization over systematic errors. The lighter area shows the additional region that would have been excluded if systematic errors had been ignored. Reprinted figure with permission from B P Abbott et al 2009 Phys. Rev. D 79122001 [73]. Copyright (2009) by the American Physical Society.

This upper limit is still significantly higher than recent CBC rate estimates derived from the observed BNS populationapproximately $5 \times 10^{-5} \mathrm{yr}^{-1} L_{10}^{-1}$ for NS/NS binaries [75].

Since the LIGO sensitivities improved as S5 progressed, analysis of the full data set should provide significantly more interesting coalescence results. In the meantime, the astrophysical reach for these sources can be estimated from the detector noise performance. The minute-by-minute strain noise spectra for each detector are used to calculate the horizon distance: the maximum distance at which an inspiral could be detected with an SNR of 8 . For BNS inspirals, the horizon distance was 30-35 Mpc each for $\mathrm{L} 1$ and $\mathrm{H} 1$ and about $17 \mathrm{Mpc}$ for $\mathrm{H} 2$. Based on the increased horizon distances and extrapolations from the first-year search results, we expect to achieve better than a factor of 2 increase in cumulative exposure with the full run analysis.

The sensitivity to $\mathrm{BH}$ ringdowns is similarly estimated using the S5 detector strain noise. Figure 11 shows the single detector range for $\mathrm{BH}$ ringdowns averaged over sky position and spin orientation. The range estimate assumes $1 \%$ of total mass is radiated as GWs, in rough agreement with numerical simulations. Unlike NS inspirals, the abundance of such 'intermediate mass BHs' and hence their merger rate is difficult to predict [62].

Searches are also in progress for GWs from CBCs with total masses up to 100 solar masses, and from CBCs coincident with short-hard GRBs observed during the S5 run. In addition, procedures are being developed for establishing confidence in candidate detection events and for extracting the physical parameters of detected events.

\subsection{GW bursts}

In addition to the well-modeled signals described in previous sections, we search for GW 'bursts', defined as any shortduration signal $(t \lesssim 1 \mathrm{~s})$ with significant signal power in the detectors' sensitive frequency band $(45 \leqslant f \leqslant 2000 \mathrm{~Hz})$. For example, the collapsing core of a massive star (the engine that

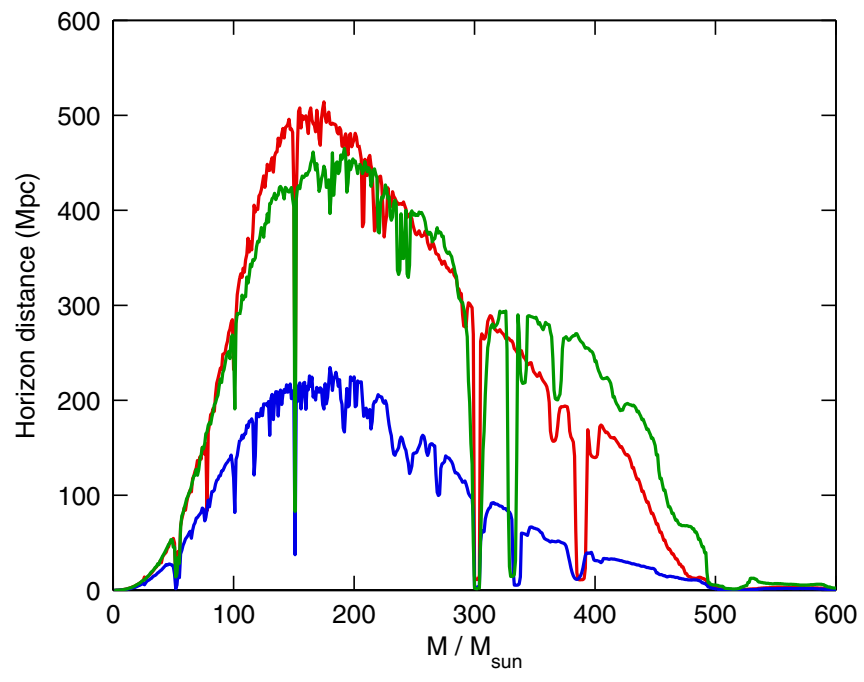

Figure 11. S5 sensitivity to binary BH ringdowns for the H1 (red), $\mathrm{L} 1$ (green) and H2 (blue) detectors. When the ringdown frequency coincides with a spectral line the sensitivity is much reduced $\left(300 M / M_{\text {sun }}\right.$ corresponds to $\left.60 \mathrm{~Hz}\right)$.

powers a type II supernova) can emit GWs through a number of different mechanisms [76]. A compact binary mergerdiscussed in the earlier section about CBC searches-may be considered a burst, especially if the mass is large so that the bulk of the long inspiral signal is below the sensitive frequency band of the detectors, leaving only a short signal from the actual merger to be detected. Cosmic strings, if they exist, are generically expected to bend into cusps and kinks which are efficient radiators of beamed GWs. There may well be other astrophysical sources, since any energetic event that involves an asymmetric reshaping or re-orientation of a significant amount of mass will generate GWs.

Many energetic gravitational events will also emit electromagnetic radiation and/or energetic particles that can be observed with telescopes and other astronomical instruments, as in the case of supernovae. Thus, besides searching for $\mathrm{GW}$ 
signals alone, we can search for a class of joint emitters and use information from conventional observations to constrain the GW event time and sky position, allowing a more sensitive 'externally triggered' search. For example, GRBs and soft gamma-ray repeater (SGR) flares are highly energetic events that make excellent targets for externally triggered GW burst searches. While the progenitor(s) of GRBs are not entirely clear, most if not all short-duration GRBs are thought to be produced by mergers of NSs or of a NS with a BH, which would radiate a great deal of energy in GWs. Similarly, SGRs are believed to be NSs with very high magnetic fields (i.e. magnetars) that sporadically produce flares of electromagnetic radiation. The flares may be related to deformations of the NS crust which could couple to GW emission. If an associated GW signal for these progenitors is detected, the combined GW and $\mathrm{EM} /$ particle data will reveal complementary information about the astrophysics of the event.

7.2.1. Analysis methods. A number of robust burst detection methods have been developed that do not rely on knowledge of the signal waveform. Most fit into one of three general categories: excess power, cross-correlation or coherent.

Excess power methods decompose the data into different frequency components, either with a Fourier basis or with some family of wavelets, and look for signal power that is significantly above the baseline detector noise level in some time-frequency region. An excess power method typically generates triggers from each detector, and then applies a coincidence test to find consistent event candidates with excess power in two or more detectors.

Cross-correlation methods directly compare the data streams from a pair of detectors to look for a common signal within uncorrelated noise. A cross-correlation statistic is calculated by integrating over a short time window-ideally, comparable in length to the duration of the signal-with a range of relative time delays corresponding to different GW arrival directions. The cross-correlation is insensitive to the relative amplitude of the common signal in the two data streams which may be different due to the antenna response of the detectors.

Coherent methods generalize the concepts of excess power and cross-correlation to take full advantage of having three or more data streams. Detectors at each site see a different linear combination of the same two time-dependent $\mathrm{GW}$ polarization components, so a network of detectors at three sites (e.g. the two LIGO sites plus Virgo) has enough information to overdetermine the waveform and provide a consistency test for each hypothetical arrival direction. This is essentially a maximum likelihood approach on the space of possible GW signals, except that a 'regulator' or Bayesian prior is used to disfavor physically unlikely scenarios $[77,78]$. If only two sites are available, the use of this regulator allows a somewhat weaker coherent analysis to be performed on data from only two detectors. In externally triggered searches, coherent analysis is simpler because the sky location of the potential signal is already known. In this case two sites are sufficient to fully determine the GW signal.

Each of these analysis methods produces a statistic (or more than one) that describes the 'strength' of the event candidate. The strength statistics are compared with the background distribution using time shift analysis, like the CBC searches. Externally triggered searches also determine the background from time shifted off-source data.

These search methods generally work well for a wide range of signals, with some waveform-dependent variation between methods. They are less sensitive than matched filtering for a known signal but are computationally efficient and are often within a factor of 2 in sensitivity.

7.2.2. Analysis results: all-sky burst searches. The most general searches are those that look for GW bursts coming from any sky position at any time. Because there is no morphological distinction between a GW burst signal and an instrumental glitch, these 'all-sky' searches place stringent demands on data quality evaluation, instrumental veto conditions and consistency tests among detectors. The primary S4 all-sky burst search [79] was designed to detect signals with frequency content in the range $64-1600 \mathrm{~Hz}$ and durations of up to $\sim 1 \mathrm{~s}$. It identified event candidates with an analysis pipeline consisting of two stages. First, a wavelet-based excess power method was used to find instances of coincident excess power in all three detectors. Second, candidate triggers were generated with highly significant correlation compared with background as well as positive definite correlation and strain amplitude between the two Hanford detectors. No significant event candidates were identified in 15.53 days of observation; based on this, we placed an upper limit at $90 \%$ confidence on the rate of detectable $\mathrm{GW}$ bursts of 0.15 per day.

To interpret a null result such as this one, a Monte Carlo method evaluates what signals could have been detected by the search. The data are re-processed with simulated GW signals using the same analysis pipeline to measure the detection efficiency in the presence of actual detector noise. The intrinsic amplitude of a simulated burst signal is characterized by a model-independent quantity, the 'root-sum-square' GW strain, $h_{\text {rss }}$, that expresses the amplitude of the GW signal arriving at the Earth without regard to the response of any particular detector. It has units of $\mathrm{Hz}^{-1 / 2}$, allowing it to be directly related to the amplitude spectral density of the detector noise as shown in figure 12 .

In principle, the efficiency of a burst search pipeline can be evaluated for any modeled GW waveform, e.g. from a core collapse simulation or a binary merger signal generated using numerical relativity. In practice, the search efficiency is evaluated for a collection of ad hoc waveforms that have certain general features but do not correspond to any particular physical model. One of our standard waveforms is a 'sineGaussian', a sinusoidal signal with central frequency $f$ within a Gaussian envelope with dimensionless width parameter $Q$. Evaluating the detection efficiency as a function of frequency, figure 12 shows the effective rate limit as a function of signal strength using an 'exclusion diagram'.

To understand the reach of the analysis in astrophysical terms, the search sensitivity in terms of $h_{\text {rss }}$ can be related to a corresponding energy emitted in GWs, $E_{\mathrm{GW}}$. As discussed in the S4 all-sky burst search paper [79], for sine-Gaussians and 


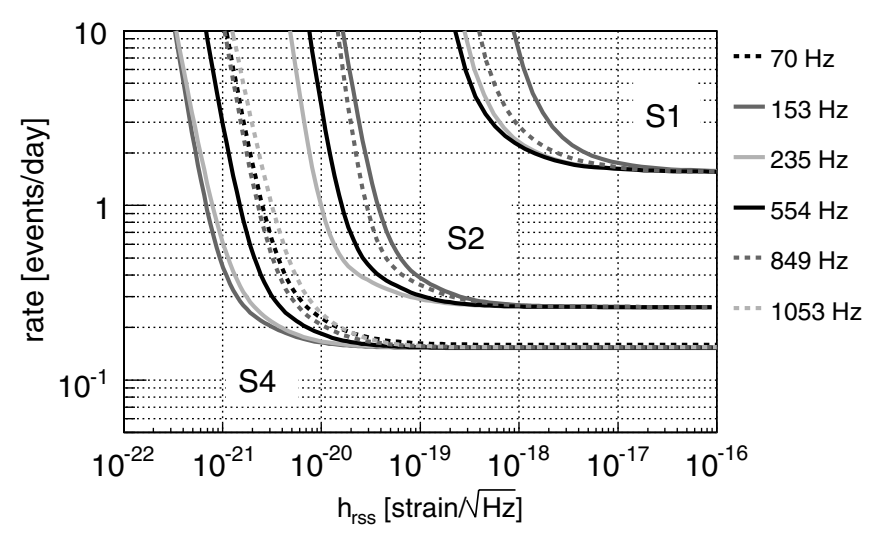

Figure 12. Exclusion diagram (rate limit at $90 \%$ confidence level, as a function of signal amplitude) for sine-Gaussian signals with $Q=8.9$. Search results from the $\mathrm{S} 1, \mathrm{~S} 2$ and $\mathrm{S} 4$ science runs are shown. (A burst search was also performed for S3, but it used only 8 days of data and systematic studies were not carried through to produce a definitive rate limit.)

other quasi-periodic signals,

$$
E_{\mathrm{GW}} \sim \frac{r^{2} c^{3}}{4 G}(2 \pi f)^{2} h_{\mathrm{rss}}^{2},
$$

where the GW energy emission is assumed to be isotropic. $\mathrm{GW}$ emission is not isotropic, but the energy flux varies by a factor of no more than 4 . Using the fact that the S5 data has lower noise than S4 by approximately a factor of 2, sources at a typical Galactic distance of $10 \mathrm{kpc}$ could be detected for energy emission in GWs as low as $\sim 5 \times 10^{-8} M_{\odot}$. For a source in the Virgo galaxy cluster, approximately $16 \mathrm{Mpc}$ away, GW energy emission as low as $\sim 0.12 M_{\odot}$ could be detected.

We can draw more specific conclusions about detectability for models of astrophysical sources that predict the absolute energy and waveform emitted. Following the discussion in [79], we estimate that a similar burst search using S5 data could detect the core-collapse signals modeled by Ott et al [80] out to $0.4 \mathrm{kpc}$ for their $11 M_{\odot}$ non-spinning progenitor (model s $11 \mathrm{WW}$ ) and to $16 \mathrm{kpc}$ for their $15 M_{\odot}$ spinning progenitor (model m15b6). The latter of these would be detectable throughout most of our Galaxy. A merger of two $10 M_{\odot}$ BHs would be detectable out to a distance of approximately $3 \mathrm{Mpc}$, while a merger of two $50 M_{\odot}$ BHs could be detected as far away as $\sim 120 \mathrm{Mpc}$.

\subsubsection{Analysis results: externally triggered burst searches.} The exceptionally intense GRB 070201 was a particularly interesting event for a triggered burst search because the sky position, determined from the gamma-ray data, overlapped one of the spiral arms of the large, nearby galaxy M31 (Andromeda). An analysis of GW data [81] found no evidence of an inspiral or a more general burst signal; that finding ruled out (at the $~ 99 \%$ level) the possibility of a binary merger in M31 being the origin of GRB 070201.

We have searched for GW bursts associated with the giant flare of SGR 1806 - 20 that occurred on 27 December 2004 (between the S4 and S5 runs, but at a time when the LIGO H1 detector was operating, albeit with reduced sensitivity) plus
190 smaller flares of SGRs $1806-20$ and $1900+14$ during the S5 run [82]. No GW signals were identified. Energy emission limits were established for a variety of hypothetical waveforms, many of them within the energy range allowed by some models and some as low as $3 \times 10^{38} \mathrm{~J}$. Future observationsespecially for giant flares and flares of the recently discovered SGR $0501+4516$ which is closer to Earth-will be sensitive to GW energy emission at or below the level of observed electromagnetic energies.

Externally triggered GW burst searches are in progress or planned using observations of supernovae, anomalous optical transients, radio bursts and neutrinos as triggers. In general, the constraints on event time, sky position and (possibly) signal properties provided by the external triggers make these searches a few times more sensitive in amplitude than all-sky searches. It is thus possible to investigate a rich population of energetic transient events that may plausibly produce detectable GWs.

\subsection{Continuous wave sources}

Continuous GW signals may be generated by rotating NSs such as those powering millisecond radio pulsars. In these systems, a quadrupole mass asymmetry, or ellipticity, $\epsilon$, radiates GWs at twice the NS rotation frequency. The maximum sustainable ellipticity, and hence the maximum $\mathrm{GW}$ emission, is a function of the NSs internal structure and equation of state. Current limits on the ellipticity are based on the change in frequency of the radio pulsar signal, the spin-down rate, assuming that the spin-down is entirely due to GW emission. An especially interesting candidate is the Crab pulsar, for which the spindown bound on ellipticity is $\epsilon \leqslant 7.2 \times 10^{-4}$ and for which the bound on detectable strain is $h \leqslant 1.4 \times 10^{-24}$ at about $59.6 \mathrm{~Hz}$, twice its spin frequency [83]. 'Standard' NS equations of state predict $\epsilon \leqslant 10^{-7}$, while exotic pulsars such as quark stars may have $\epsilon \leqslant 10^{-4}$ [84]. For most radio pulsars, however, the spindown limit overestimates the ellipticity and associated $\mathrm{GW}$ emission because of electromagnetic damping of the rotation.

Compared with CBCs or bursts, NS powered millisecond radio pulsars are a weak source of GWs which LIGO can detect only if the source is within a few hundred parsecs. Nonetheless, there are dozens of known sources within this range that may be detected if they have sufficiently high ellipticity. Furthermore, millisecond pulsars are attractive sources of continuous GWs since the stable rotation periods allow coherent integration over many hours, weeks and months to improve the SNR.

7.3.1. Analysis methods. The detectable waveform from a rotating NS is a function of at least six source parameters: two each for the pulsar position and orientation on the sky and at least two for the spin frequency and frequency drift (1st time derivative). The intrinsic phase of a spinning NS waveform, as measured in the NS's rest frame, $\Phi(T)$, is modeled as an approximate sinusoid at instantaneous frequency $v$ and spin-down rate $\dot{v}$. The observed phase in the detector frame, $\phi(t)$, is in general a more complicated function of time due to the variable time delay $\delta t=T-t$. The delay $\delta t$ contains components arising from the Earth's orbital motion 
(for which $|\delta t| \leqslant 8.5 \mathrm{~min}$ ), from the Earth's sidereal motion $(|\delta t| \leqslant 43 \mu \mathrm{s})$ and from the general relativistic Shapiro delay $(|\delta t| \leqslant 120 \mu \mathrm{s})$ for signals passing close to the sun [85].

The six-dimensional parameter space and long duration of the S5 run makes all-sky coherent searches for unknown NSs, for which the amplitude and phase variations are tracked throughout the observation time, computationally prohibitive. Three techniques that trade off between sensitivity and computation have been implemented: (1) semi-coherent, long duration all-sky searches sensitive only to power and neglecting phase using the entire data set [86]; (2) coherent, short-duration all-sky searches sensitive to amplitude and phase but computationally limited to $\approx 5000 \mathrm{~h}$ integration time [87]; (3) coherent, targeted searches for millisecond radio pulsars with accurate and stable ephemerides using the entire data set [88]. Although the coherent targeted search is the most sensitive, only a little more than 100 known radio pulsars have suitable ephemerides, while NS formation rates predict that many hundred millisecond pulsars should exist within a detectable volume [89]. Thus even though the all-sky searches are not as sensitive as the targeted search, they are worth performing.

The LIGO Scientific Collaboration has implemented several different incoherent all-sky searches. The most recent results using the S5 data are from PowerFlux [89]. The search averages strain power from short Fourier transforms (SFTs) over the full run to look for excess power in a narrow frequency bin. The SFTs are calculated using contiguous 30 min data segments. Before summing, each SFT is shifted by a sky position-dependent factor to account for the time delays discussed above, and weighted according to the detector antenna response and average noise power. Frequency bins with high SNR are checked for coincidence between multiple detectors and followed up with coherent searches.

An alternative all-sky search using longer coherence times ( $>1$ day) offers improved sensitivity, but its computational demands require a new paradigm: distributed computing using the Einstein@Home network [90]. Einstein@Home users volunteer their idle computing CPU cycles to perform a coherent analysis. The combined resources of 50000 volunteers with 100000 CPUs enables an all-sky search for rotating NSs in $5280 \mathrm{~h}$ of the most sensitive S5 data. The Einstein@Home search is based on the coherent $\mathcal{F}$-statistic in the frequency domain [54]. Each CPU in the distributed network calculates the coherent signal for each frequency bin and sky position for a $30 \mathrm{~h}$ contiguous segment. The loudest frequency bins are followed up with coincidence studies between detectors and continuity studies with adjacent time segments.

The deepest searches are performed for millisecond radio pulsars with well-characterized, stable ephemerides. The 154 pulsars with spin frequencies greater than $25 \mathrm{~Hz}$ are selected from the Australian Telescope National Facility online catalogue [91]. Of these, 78 have sufficient stability and timing resolution to make knowledge of their waveform capable of improving the detection SNR over the long observation time. To consistently incorporate the prior information, the targeted search uses a time-domain, Bayesian analysis in

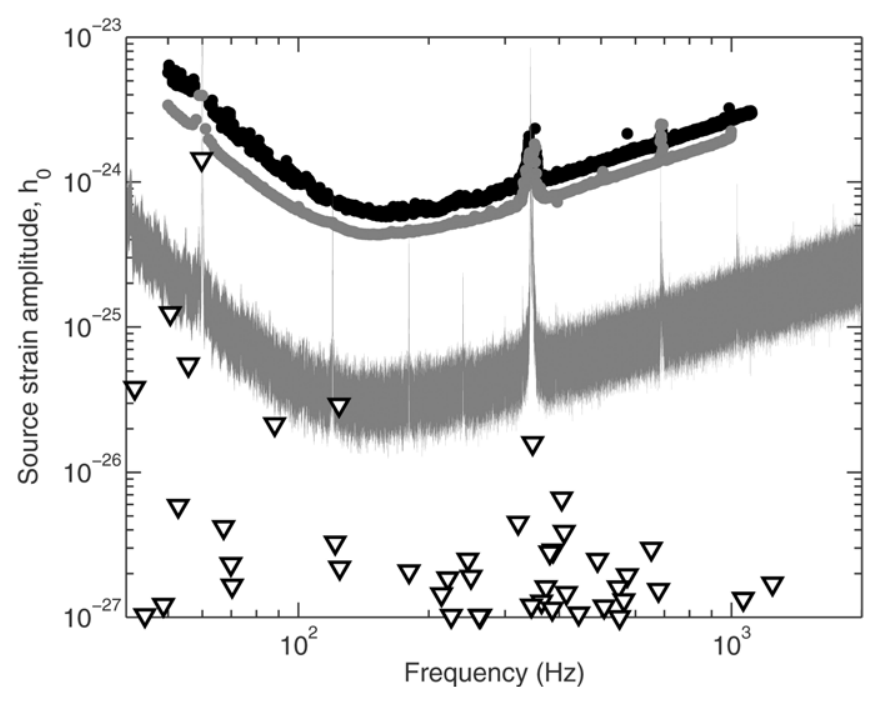

Figure 13. Limits on GW strain from rotating NSs. Upper curve (black points): all-sky strain upper limits on unknown NSs for spin-down rates as high as $5 \times 10^{-9} \mathrm{~Hz} \mathrm{~s}^{-1}$ and optimal orientation, from analysis of the first 8 months of S5 data [89]. Middle curve (gray points): expected sensitivity for the Einstein@Home search with $5280 \mathrm{~h}$ of S5 data. Lower curve (gray band): expected range for 95\% confidence level Bayesian upper limits on radio pulsars with known epherimides, using the full S5 data. Black triangles: upper limits on GW emission from known radio pulsars based on their observed spin-down rates.

which the detection likelihood is calculated for each detector. Information from multiple detectors is combined to form a joint likelihood assuming the detectors' noises are independent. This procedure allows upper limits from successive science runs to be combined and provides a natural framework for incorporating uncertainties in the ephemerides.

7.3.2. Analysis results. Analyses of the full S5 data are underway using the techniques described above. An all-sky search using the PowerFlux technique on the first 8 months of S5 with the H1 and L1 detectors has been completed [89]. This produced upper limits on strain amplitude in the band $50-1100 \mathrm{~Hz}$. For a NS with equatorial ellipticity of $10^{-6}$, the search was sensitive to distances as great as $500 \mathrm{pc}$.

Because of the narrow bandwidth $\left(10^{-6} \mathrm{~Hz}\right)$ and complicated frequency modulation of pulsar signals, instrument artifacts do not significantly contribute to the noise in pulsar searches. The few exceptions-non-stationary noise near $60 \mathrm{~Hz}$ harmonics, wandering lines, etc-have been easy to identify and remove. Consequently, we can predict the astrophysical reach of the full S5 data set with a high degree of confidence based on the performance of previous searches and the S5 noise performance. Figure 13 shows the projected S5 strain amplitude sensitivity for the more sensitive searches, along with the upper limits established by the PowerFlux analysis.

\subsection{Stochastic GW background}

A stochastic background of GWs could result from the random superposition of an extremely large number of unresolved and 
independent GW emission events [92]. Such a background is analogous to the cosmic microwave background radiation, though its spectrum is unlikely to be thermal. The emission events could be the result of cosmological phenomena, such as the amplification of vacuum fluctuations during inflation or in pre-big-bang models; phase transitions in the history of the universe or cosmic strings, topological defects that may have been formed during symmetry-breaking phase transitions in the early universe. Or a detectable background could result from many unresolved astrophysical sources, such as rotating NSs, supernovae or low-mass $\mathrm{x}$-ray binaries.

Theoretical models of such sources are distinguished by the power spectra they predict for the stochastic background production. The spectrum is usually described by the dimensionless quantity $\Omega_{\mathrm{GW}}(f)$, which is the $\mathrm{GW}$ energy density per unit logarithmic frequency, divided by the critical energy density $\rho_{\mathrm{c}}$ to close the universe:

$$
\Omega_{\mathrm{GW}}(f)=\frac{f}{\rho_{\mathrm{c}}} \frac{\mathrm{d} \rho_{\mathrm{GW}}}{\mathrm{d} f} .
$$

In the LIGO frequency band, most of the model spectra are well approximated by a power-law: $\Omega_{\mathrm{GW}}(f) \propto f^{\alpha}$. LIGO analyses consider a range of values for $\alpha$, though in this review we focus on results for a frequency independent $\Omega_{\mathrm{GW}}(\alpha=0)$, since many of the cosmological models predict a flat or nearly flat spectrum over the LIGO band. The strain noise power spectrum for a flat $\Omega_{\mathrm{GW}}$ falls as $f^{-3}$, with a strain amplitude scale of ${ }^{64}$ :

$$
h_{\mathrm{GW}}=4 \times 10^{-22} \sqrt{\Omega_{\mathrm{GW}}}\left(\frac{100 \mathrm{~Hz}}{f}\right)^{3 / 2} \mathrm{~Hz}^{-1 / 2} .
$$

7.4.1. Analysis method. Unlike the cosmic microwave background, any GW stochastic background will be well below the noise floor of a single detector. To probe below this level, we cross-correlate the output of two detectors [93]. Assuming the detector noises are independent of each other, in the cross-correlation measurement the signal-due to a stochastic background present in each output-will increase linearly with integration time $T$, whereas the measurement noise will increase only with the square root of $T$. Similarly the signal will increase linearily with the effective bandwidth $(\Delta f)$ of the correlation and the noise as $(\Delta f)^{1 / 2}$. Thus with a sufficiently long observation time and wide bandwidth, a small background signal can, in principle, be detected beneath the detector noise floor.

The assumption of independent detector noise is crucial, and it is valid when comparing L1 with either of the Hanford detectors due to their wide physical separation. But this separation also extracts a price: the coherent crosscorrelation of a stochastic background signal is reduced by the separation time delay between the detectors and the nonparallel alignment of their arms. These effects are accounted for by the overlap reduction function $\gamma(f)$, which is unity for co-located and co-aligned detectors, and decreases below unity

${ }^{64}$ We assume here and in the rest of this paper a Hubble expansion rate of $72 \mathrm{~km} / \mathrm{s} / \mathrm{Mpc}$. when they are shifted apart or mis-aligned. For a LivingstonHanford detector pair, the overlap is on average $\langle\gamma\rangle \sim 0.1$ in the sensitive band around $100 \mathrm{~Hz}$.

The low-frequency noise floor of a single S5 LIGO detector is roughly equivalent to a stochastic background spectrum with $\Omega_{\mathrm{GW}}=0.01\left(h_{\mathrm{GW}}=4 \times 10^{-23} \mathrm{~Hz}^{-1 / 2}\right.$ at $100 \mathrm{~Hz}$ ). The cross-correlation measurement will be sensitive to a background $\Omega_{\mathrm{GW}}$ lower than this noise floor by a factor of $\langle\gamma\rangle(T \Delta f)^{1 / 2}$. With a year of observation time and an effective bandwidth of $100 \mathrm{~Hz}$ this is a factor of several thousand, so we expect to probe for a stochastic background in the range $\Omega_{\mathrm{GW}} \sim 10^{-5}-10^{-6}$.

7.4.2. Analysis results. Since the SNR for a search on $\Omega_{\mathrm{GW}}$ grows inversely with the product of the strain noise amplitude spectra of the two detectors, the sensitivity of this search grew quickly as the detectors improved. Analysis of the S4 data yielded a Bayesian $90 \%$ upper limit of $\Omega_{\mathrm{GW}}<6.5 \times 10^{-5}$ for a flat spectrum in the $51-150 \mathrm{~Hz}$ band [94]. Projecting for the S5 data, the lower strain noise and longer integration time should improve on this by an order of magnitude. While the cross-correlation analysis for S5 is still in progress, it is straightforward to calculate the expected variance of the crosscorrelation using only the interferometers' strain noise spectra over the run. This predicts that the potential upper limit on $\Omega_{\mathrm{GW}}$ will be in the range $(4-5) \times 10^{-6}$.

Such a result would be the first direct measurement to place a limit on $\Omega_{\mathrm{GW}}$ more stringent than the indirect bound set by big-bang-nucleosynthesis (BBN). The BBN bound, currently the most constraining bound in the band around $100 \mathrm{~Hz}$, derives from the fact that a large GW energy density present at the time of BBN would have altered the abundances of the light nuclei in the universe [55,92]. For the BBN model to be consistent with observations of these abundances, the total GW energy density at the time of nucleosynthesis is thus constrained. In the limiting case that the GW energy was confined to LIGO's sensitive band of $50-150 \mathrm{~Hz}$, the BBN bound is $\Omega_{\mathrm{GW}}<1.1 \times 10^{-5}[55,95]$.

The LIGO results are also being used to constrain the parameter space of models predicting a stochastic GW background, such as cosmic string models and pre-bigbang models [94]. The gamut of theoretical models and observations pertaining to a stochastic GW background spans an impressively wide range of frequencies and amplitudes. These are displayed in the landscape plot of figure 14 .

The analysis described so far searches for an isotropic background of GWs. The cross-correlation method has also been extended to search for spatial anisotropies, as might be produced by an ensemble of astrophysical sources [96]. Such a $\mathrm{GW}$ radiometer requires spatially separated interferometers in order to point the multi-detector antenna at different locations on the sky. The result is a map of the power distribution of GWs convolved with the antenna lobe of the radiometer, with an uncertainty determined by the detector noise. Radiometer analysis of the S4 data yielded upper limits on the GW strain power from point sources in the range of $\sim 10^{-48}$ $\sim 10^{-47} \mathrm{~Hz}^{-1}$, depending on sky position and the GW power spectrum model [97]. The S5 analysis should improve on the 


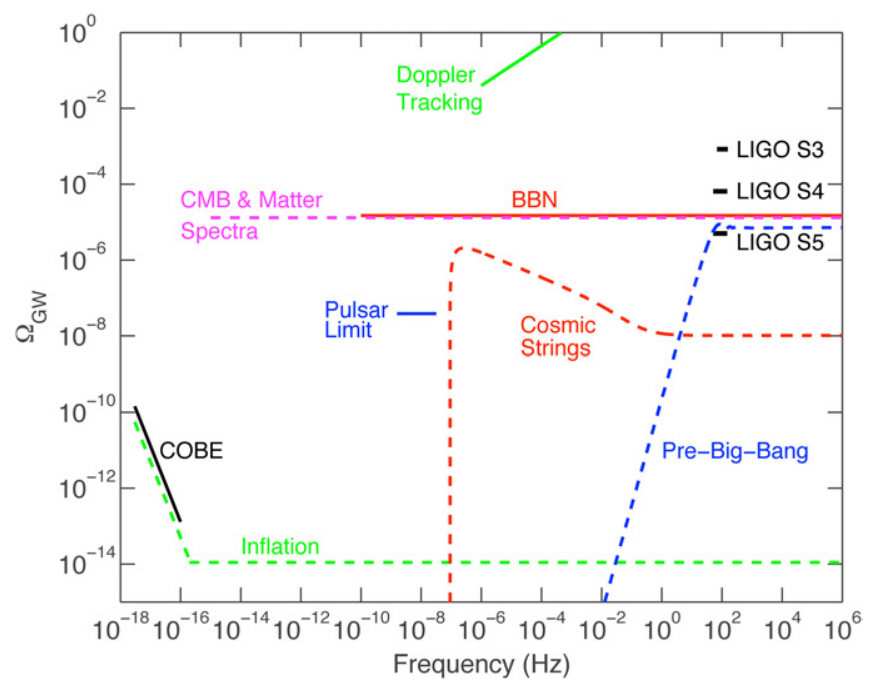

Figure 14. Observational limits and potential sources for a stochastic background of GWs. The LIGO S5 curve refers to the potential upper limit from the S5 run, based on strain noise data. The curves corresponding to inflationary, cosmic-string and pre-big-bang models are examples; the model parameters allow significant variation in the predicted spectra. The BBN and CMB \& Matter Spectra bounds apply to the total GW energy over the frequency range spanned by the corresponding lines. See [94] for more details.

strain power sensitivity by a factor of 30 . The corresponding GW energy flux density that the search will be sensitive to is $\sim 10^{-10} \mathrm{~W} / \mathrm{m}^{2} / \mathrm{Hz}(f / 100 \mathrm{~Hz})^{2}$.

\section{Future directions}

From its inception, LIGO was envisioned not as a single experiment, but as an on-going observatory. The facilities and infrastructure construction were specified, as much as possible, to accommodate detectors with much higher sensitivity. We have identified a set of relatively minor improvements to the current instruments [98] that can yield a factor of 2 increase in strain sensitivity and a corresponding factor of 8 increase in the probed volume of the universe. The two most significant enhancements are higher laser power and a new, more efficient readout technique for the $\mathrm{GW}$ channel. Higher power is delivered by a new master oscillator-power amplifier system, emitting $35 \mathrm{~W}$ of single frequency $1064 \mathrm{~nm}$ light [99], 3.5 times the power of the initial LIGO lasers. For the readout, a small mode-cleaner cavity is inserted in the AS beam path, between the Faraday isolator and the length photodetectors. This cavity filters out RF sidebands and the higher order mode content of the AS port light, reducing the shot-noise power. Instead of RF heterodyning, signal detection is done by slightly offsetting the differential arm length from the dark fringe, and using the resulting carrier field as the local oscillator in a dc homodyne detection scheme. These improvements (known collectively as Enhanced LIGO) are currently being implemented and commissioned on $\mathrm{H} 1$ and $\mathrm{L} 1$, and a 1-2 year science run with these interferometers is expected to begin in mid-2009.

Significantly greater sensitivity improvements are possible with more extensive upgrades. Advanced LIGO will replace the existing interferometers with significantly improved technology to achieve a factor of at least 10 in sensitivity over the initial LIGO interferometers and to lower the seismic wall frequency down to $10 \mathrm{~Hz}[100,101]$. Advanced LIGO has been funded by the National Science Foundation, beginning in April 2008. Installation of the Advanced LIGO interferometers is planned to start in early 2011.

The Advanced LIGO interferometers are configured like initial LIGO - a power-recycled Fabry-Perot Michelsonwith the addition of a signal recycling mirror at the antisymmetric output. Signal recycling gives the ability to tune the interferometer frequency response, so that the point of maximum response can be shifted away from zero frequency [9]. The laser wavelength stays at $1064 \mathrm{~nm}$, but an additional high-power stage brings the laser power up to $200 \mathrm{~W}$ [102]. The test masses will be significantly larger $-40 \mathrm{~kg}$ - in order to reduce radiation pressure noise and to allow larger beam sizes. Larger beams and better dielectric mirror coatings combine to reduce the test mass thermal noise by a factor of 5 compared with initial LIGO [103].

The test mass suspensions become significantly more intricate to provide much better performance. They incorporate four cascaded stages of passive isolation, instead of just one, including vertical isolation comparable to the horizontal isolation at all stages except one [104]. The test mass is suspended at the final stage with fused-silica fibers rather than steel wires; these fibers have extremely low mechanical loss and will reduce suspension thermal noise nearly a hundred-fold [105]. The current passive seismic isolation stacks that support the suspensions are replaced with two-stage active isolation platforms [106]. These stages are designed to actively reduce the ground vibration by a factor of $\sim 1000$ in the $1-10 \mathrm{~Hz}$ band, and provide passive isolation at higher frequencies. The combination of the isolation platforms and the suspensions will reduce seismic noise to negligible levels above approximately $10 \mathrm{~Hz}$.

The successful operation of Advanced LIGO is expected to transform the field from $\mathrm{GW}$ detection to $\mathrm{GW}$ astrophysics. We illustrate the potential using compact binary coalescences. Detection rate estimates for CBCs can be made using a combination of extrapolations from observed binary pulsars, stellar birth rate estimates and population synthesis models. There are large uncertainties inherent in all of these methods, however, leading to rate estimates that are uncertain by several orders of magnitude. We therefore quote a range of rates, spanning plausible pessimistic and optimistic estimates, as well as a likely rate. For a NS mass of $1.4 M_{\odot}$ and a $\mathrm{BH}$ mass of $10 M_{\odot}$, these rate estimates for Advanced LIGO are: 0.4$400 \mathrm{yr}^{-1}$, with a likely rate of $40 \mathrm{yr}^{-1}$ for NS-NS binaries; $0.2-300 \mathrm{yr}^{-1}$, with a likely rate of $10 \mathrm{yr}^{-1}$ for NS-BH binaries; $2-4000 \mathrm{yr}^{-1}$, with a likely rate of $30 \mathrm{yr}^{-1}$ for $\mathrm{BH}-\mathrm{BH}$ binaries.

\section{Acknowledgments}

The authors gratefully acknowledge the support of the United States National Science Foundation for the construction and operation of the LIGO Laboratory and the Particle Physics 
and Astronomy Research Council of the UK, the Max-PlanckSociety and the State of Niedersachsen/Germany for support of the construction and operation of the GEO 600 detector. The authors also gratefully acknowledge the support of the research by these agencies and by the Australian Research Council, the Natural Sciences and Engineering Research Council of Canada, the Council of Scientific and Industrial Research of India, the Department of Science and Technology of India, the Spanish Ministerio de Educacion y Ciencia, The National Aeronautics and Space Administration, the John Simon Guggenheim Foundation, the Alexander von Humboldt Foundation, the Leverhulme Trust, the David and Lucile Packard Foundation, the Research Corporation and the Alfred P Sloan Foundation.

\section{References}

[1] Weisberg J M and Taylor J H 2005 The relativistic binary pulsar B1913 + 16: thirty years of observations and analysis Binary Radio Pulsars ed F A Rasio and I H Stairs (Astronomical Society of the Pacific Conference Series vol 328) (San Francisco, CA: Astronomical Society of the Pacific) pp 25-31

[2] Abramovici A et al 1992 LIGO: the Laser Interferometer Gravitational-Wave Observatory Science 256 325-33

[3] Cutler C and Thorne K S 2002 An overview of gravitational-wave sources arXiv:gr-qc/0204090v1

[4] Homepage of the LIGO Scientific Collaboration http://www.ligo.org

[5] Takahashi R 2004 Status of TAMA300 Class. Quantum Grav. 21 S403-8

[6] Luck H et al 2006 Status of the GEO600 detector Class. Quantum Grav. 23 S71-8

[7] Acernese F et al 2006 The Virgo status Class. Quantum Grav. 23 S635-42

[8] Kuroda K and the LCGT Collaboration 2006 The status of LCGT Class. Quantum Grav. 23 S215-21

[9] Meers B J 1988 Recycling in laser interferometric gravitational wave detectors Phys. Rev. D 38 2317-26

[10] Savage R L, King P J and Seel S U 1998 A highly stabilized $10 \mathrm{~W}$ Nd: YAG laser for the Laser Interferometer Gravitational-wave Observatory (LIGO) Laser Phys. 8 679-85

[11] Abbott R S and King P J 2001 Diode-pumped Nd: YAG laser intensity noise suppression using a current shunt Rev. Sci. Instrum. 72 1346-9

[12] Drever R W P, Hall J L, Kowalski F V, Hough J, Ford G M, Munley A J and Ward H 1983 Laser phase and frequency stabilization using an optical resonator Appl. Phys. B: Lasers Opt. 31 97-105

[13] Willke B, Uehara N, Gustafson E K, Byer R L, King P J, Seel S U and Savage R L Jr 1998 Spatial and temporal filtering of a $10 \mathrm{~W} \mathrm{Nd}$ : YAG laser with a Fabry-Perot ring-cavity pre-mode cleaner Opt. Lett. 23 1704-6

[14] Zucker M E and Whitcomb S E 1996 Measurement of optical path fluctuations due to residual gas in the LIGO 40 meter interferometer Proc. 7th Marcel Grossman Meeting on Recent Developments in Theoretical and Experimental General Relativity, Gravitation, and Relativistic Field Theories ed R T Jantzen et al (Stanford, CA, USA, 24-29 July 1994) (Singapore: World Scientific) pp 1434-6

[15] Skeldon K D, Strain K A, Grant A I and Hough J 1996 Test of an 18-m-long suspended modecleaner cavity Rev. Sci. Instrum. $672443-8$

[16] Walsh C J, Leistner A J, Seckold J, Oreb B F and Farrant D I 1999 Fabrication and measurement of optics for the laser interferometer gravitational wave observatory Appl. Opt. 38 2870-9

[17] Wei D T 1989 Ion beam interference coating for ultralow optical loss Appl. Opt. 28 2813-6

[18] Rempe G, Thompson R J, Kimble H J and Lalezari R 1992 Measurement of ultralow losses in an optical interferometer Opt. Lett. 17 363-5

[19] Ottaway D, Betzwieser J, Ballmer S, Waldman S and Kells W 2006 In situ measurement of absorption in high-power interferometers by using beam diameter measurements Opt. Lett. 31 450-2

[20] Vinet J-Y, Brisson V and Braccini S 1996 Scattered light noise in gravitational wave interferometric detectors: coherent effects Phys. Rev. D 54 1276-86

[21] Vinet J-Y et al 1997 Scattered light noise in gravitational wave interferometric detectors: a statistical approach Phys. Rev. D 56 6085-95

[22] Giaime J, Saha P, Shoemaker D and Sievers L 1996 A passive vibration isolation stack for LIGO: design, modeling, and testing Rev. Sci. Instrum. 67 208-14

[23] Abbott $\mathrm{R}$ et al 2004 Seismic isolation enhancements for initial and advanced LIGO Class. Quantum Grav. 21 S915-21

[24] Fritschel P, Bork R, González G, Mavalvala N, Ouimette D, Rong H, Sigg D and Zucker M 2001 Readout and control of a power-recycled interferometric gravitational-wave antenna Appl. Opt. 40 4988-98

[25] Regehr M W, Raab F J and Whitcomb S E 1995 Demonstration of a power-recycled Michelson interferometer with Fabry-Perot arms by frontal modulation Opt. Lett. 20 1507-9

[26] Adhikari R 2004 Sensitivity and noise analysis of $4 \mathrm{~km}$ laser interferometric gravitational wave antennae $P h D$ Thesis MIT

[27] Morrison E, Robertson D I, Ward H and Meers B J 1994 Automatic alignment of optical interferometers Appl. Opt. 33 5041-9

[28] Hefetz Y, Mavalvala N and Sigg D 1997 Principles of calculating alignment signals in complex resonant optical interferometers J. Opt. Soc. Am. B: Opt. Phys. 14 1597-605

[29] Fritschel P, Mavalvala N, Shoemaker D, Sigg D, Zucker M and González G 1998 Alignment of an interferometric gravitational wave detector Appl. Opt. 37 6734-47

[30] Evans M et al 2002 Lock acquisition of a gravitational-wave interferometer Opt. Lett. 27 598-600

[31] Winkler W, Danzmann K, Rüdiger A and Schilling R 1991 Heating by optical absorption and the performance of interferometric gravitational-wave detectors Phys. Rev. A $447022-36$

[32] D'Ambrosio E and Kells W 2006 Carrier mode selective working point and side band imbalance in LIGO I Phys. Rev. D 73122002

[33] Gretarsson A M, D'Ambrosio E, Frolov V, O'Reilly B and Fritschel P K 2007 Effects of mode degeneracy in the LIGO Livingston Observatory recycling cavity J. Opt. Soc. Am. B 24 2821-8

[34] Ballmer S et al 2005 Thermal compensation system description Technical Report LIGO-T050064-00 LIGO Project

[35] Landry M and the LIGO Scientific Collaboration 2005 Improvements in strain calibration for the third LIGO science run Class. Quantum Grav. 22985

[36] Meers B J 1989 The frequency response of interferometric gravitational wave detectors Phys. Lett. A 142 465-70

[37] Del Fabbro R and Montelatici V 1995 Interferometric antenna response for gravitational-wave detection Appl. Opt. 34 4380-96 
[38] Rakhmanov M, Romano J D and Whelan J T 2008 High-frequency corrections to the detector response and their effect on searches for gravitational waves Class. Quantum Grav. 25184017

[39] Niebauer T M, Schilling R, Danzmann K, Rüdiger A and Winkler W 1991 Nonstationary shot noise and its effect on the sensitivity of interferometers Phys. Rev. A 43 5022-9

[40] Sigg D 1997 Frequency response of the LIGO interferometer Technical Report LIGO-T970084-00 LIGO Project

[41] Camp J B, Yamamoto H, Whitcomb S E and McClelland D E 2000 Analysis of light noise sources in a recycled Michelson interferometer with Fabry-Perot arms J. Opt. Soc. Am. A 17 120-8

[42] Saulson P R 1990 Thermal noise in mechanical experiments Phys. Rev. D 42 2437-45

[43] Levin Y 1998 Internal thermal noise in the LIGO test masses: a direct approach Phys. Rev. D 57 659-63

[44] Gonzalez G 2000 Suspensions thermal noise in the LIGO gravitational wave detector Class. Quantum Grav. 17 4409-35

[45] Harry G M et al 2002 Thermal noise in interferometric gravitational wave detectors due to dielectric optical coatings Class. Quantum Grav. 19 897-917

[46] Gillespie A and Raab F J 1994 Suspension losses in the pendula of laser interferometer gravitational wave detectors Phys. Lett. A 190 213-20

[47] Cagnoli G, Gammaitoni L, Kovalik J, Marchesoni F and Punturo M 1999 Low-frequency internal friction in clamped-free thin wires Phys. Lett. A 255 230-5

[48] Penn S D, Ageev A, Busby D, Harry G M, Gretarsson A M, Numata K and Willems P 2006 Frequency and surface dependence of the mechanical loss in fused silica Phys. Lett. A 352 3-6

[49] Kawamura S and Zucker M E 1994 Mirror-orientation noise in a Fabry-Perot interferometer gravitational wave detector Appl. Opt. 33 3912-8

[50] Cote P J and Meisel L V 1991 Self-organized criticality and the Barkhausen effect Phys. Rev. Lett. 671334

[51] Blackburn L et al 2008 The LSC glitch group: monitoring noise transients during the fifth LIGO science run Class. Quantum Grav. 25184004

[52] Belczynski K, Kalogera V and Bulik T 2001 A comprehensive study of binary compact objects as gravitational wave sources: evolutionary channels, rates, and physical properties Astrophys. J. 572 407-31

[53] Dimmelmeier H, Font J A and Muller E 2001 Gravitational waves from relativistic rotational core collapse Astrophys. J. 560 L163-6

[54] Jaranowski P, Krolak A and Schutz B F 1998 Data analysis of gravitational-wave signals from spinning neutron stars: I. The signal and its detection Phys. Rev. D 58063001

[55] Maggiore M 2000 Gravitational wave experiments and early universe cosmology Phys. Rep. 331 283-367

[56] Links to all published observational results from LIGO can be found at http://www.lsc-group.phys.uwm.edu /ppcomm/Papers.html

[57] Will C M 2006 The confrontation between general relativity and experiment Living Rev. Rel. 93

[58] Blanchet L 2006 Gravitational radiation from post-Newtonian sources and inspiralling compact binaries Living Rev. Rel. 94

[59] Tagoshi H and Sasaki M 2003 Analytic black hole perturbation approach to gravitational radiation Living Rev. Rel. 66

[60] Fox D B et al 2005 The afterglow of GRB 050709 and the nature of the short-hard $\gamma$-ray bursts Nature $437845-50$

[61] Blanchet L 2002 Gravitational radiation from post-Newtonian sources and inspiralling compact binaries Living Rev. Rel. 53
[62] Flanagan E E and Hughes S A 1998 Measuring gravitational waves from binary black hole coalescences: I. Signal to noise for inspiral, merger, and ringdown Phys. Rev. D 57 4535-65

[63] Blanchet L, Damour T, Iyer B R, Will C M and Wiseman A G 1995 Gravitational radiation damping of compact binary systems to second post-Newtonian order Phys. Rev. Lett. 74 3515-8

[64] Hannam M 2009 Status of black-hole-binary simulations for gravitational- wave detection arXiv:0901.2931 [gr-qc]

[65] Aylott B et al 2009 Testing gravitational-wave searches with numerical relativity waveforms: results from the first Numerical INJection Analysis (NINJA) project arXiv:0901.4399 [gr-qc]

[66] Creighton J D E 1999 Search techniques for gravitational waves from black-hole ringdowns Phys. Rev. D 6002200

[67] Owen B J and Sathyaprakash B S 1999 Matched filtering of gravitational waves from inspiraling compact binaries: Computational cost and template placement Phys. Rev. D 60022002

[68] Babak S, Balasubramanian R, Churches D, Cokelaer T and Sathyaprakash B S 2006 A template bank to search for gravitational waves from inspiralling compact binaries: I. Physical models Class. Quantum Grav. 23 5477-504

[69] Cokelaer T 2007 Gravitational waves from inspiralling compact binaries: hexagonal template placement and its efficiency in detecting physical signals Phys. Rev. D 76102004

[70] Abbott B et al 2008 Search for gravitational waves from binary inspirals in S3 and S4 LIGO data Phys. Rev. D 77062002

[71] Allen B 2005 A $\chi^{2}$ time-frequency discriminator for gravitational wave detection Phys. Rev. D 71062001

[72] Rodríguez A 2007 Reducing false alarms in searches for gravitational waves from coalescing binary systems Master's Thesis Louisiana State University

[73] LIGO Scientific Collaboration 2009 Search for gravitational waves from low mass binary coalescences in the first year of LIGO's S5 data Phys. Rev. D 79122001

[74] Kopparapu R K, Hanna C, Kalogera V, OShaugh nessy R, Gonzlez G, Brady P R and Fairhurst S 2008 Host galaxies catalog used in LIGO searches for compact binary coalescence events Astrophys. J. 675 1459-67

[75] Kalogera V et al 2004 The cosmic coalescence rates for double neutron star binaries Astrophys. J. 601 L179-82

[76] Ott C D 2009 The gravitational wave signature of core-collapse supernovae Class. Quantum Grav. 26063001

[77] Rakhmanov M 2006 Rank deficiency and Tikhonov regularization in the inverse problem for gravitational-wave bursts Class. Quantum Grav. 23 S673-85

[78] Cannon K C 2008 A Bayesian coincidence test for noise rejection in a gravitational-wave burst search Class. Quantum Grav. 25105024

[79] Abbott B et al 2007 Search for gravitational-wave bursts in LIGO data from the fourth science run Class. Quantum Grav. 24 5343-69

[80] Ott C D, Burrows A, Dessart L and Livne E 2006 A new mechanism for gravitational wave emission in corecollapse supernovae Phys. Rev. Lett. 96201102

[81] Abbott B and for the LIGO Scientific Collaboration 2008 Implications for the origin of GRB 070201 from LIGO observations Astrophys. J. 681 1419-28

[82] Abbott B et al 2008 Search for gravitational wave bursts from soft gamma repeaters Phys. Rev. Lett. 101211102

[83] Abbott B et al 2008 Beating the spin-down limit on gravitational wave emission from the Crab pulsar Astrophys. J. 683 L45-50 
[84] Owen B J 2005 Maximum elastic deformations of compact stars with exotic equations of state Phys. Rev. Lett. 95211101

[85] Backer D C and Hellings R W 1986 Pulsar timing and general relativity Ann. Rev. Astron. Astrophys. 24 537-75

[86] Abbott B et al 2008 All-sky search for periodic gravitational waves in LIGO S4 data Phys. Rev. D 77022001

[87] Abbott B et al 2007 Searches for periodic gravitational waves from unknown isolated sources and Scorpius X-1: results from the second LIGO science run Phys. Rev. D 76082001

[88] Abbott B et al 2007 Upper limits on gravitational wave emission from 78 radio pulsars Phys. Rev. D 76042001

[89] LIGO Scientific Collaboration 2009 All-sky LIGO search for periodic gravitational waves in the early S5 data Phys. Rev. Lett. 102111102

[90] The Einstein@Home website can be found at http://einstein.phys.uwm.edu/

[91] http://www.atnf.csiro.au/research/pulsar/psrcat/

[92] Allen B 1996 The stochastic gravity-wave background: sources and detection arXiv:gr-qc/9604033v3

[93] Allen B and Romano J D 1999 Detecting a stochastic background of gravitational radiation: signal processing strategies and sensitivities Phys. Rev. D 59102001

[94] Abbott B 2007 Searching for a stochastic background of gravitational waves with the laser interferometer gravitational-wave observatory Astrophys. J. 659 918-30

[95] Cyburt R H, Fields B D, Olive K A and Skillman E 2005 New BBN limits on physics beyond the standard model from 4He Astropart. Phys. 23 313-23

[96] Ballmer S W 2006 A radiometer for stochastic gravitational waves Class. Quantum Grav. 23 S179-85
[97] Abbott B et al 2007 Upper limit map of a background of gravitational waves Phys. Rev. D 76082003

[98] Adhikari R, Fritschel P and Waldman S 2006 Enhanced LIGO Technical Report LIGO-T060156-01 LIGO Project

[99] Frede M, Schulz B, Wilhelm R, Kwee P, Seifert F, Willke B and Kracht D 2007 Fundamental mode, single-frequency laser amplifier for gravitational wave detectors $O p t$. Express 15 459-65

[100] Fritschel P 2003 Second generation instruments for the Laser Interferometer Gravitational Wave Observatory (LIGO) Presented at the Society of Photo-Optical Instrumentation Engineers (SPIE) Conf. (Waikoloa, HI, USA, 22-28 August 2002) (Society of Photo-Optical Instrumentation Engineers (SPIE) Conference Series vol 4856) ed M Cruise and P Saulson (Bellingham, WA: SPIE Optical Engineering Press) pp 282-91

[101] Advanced LIGO team 2006 Advanced LIGO reference design Technical Report LIGO-M060056-08 LIGO Project

[102] Willke B et al 2008 Stabilized lasers for advanced gravitational wave detectors Class. Quantum Grav. 25114040

[103] Harry G M et al 2007 Titania-doped tantala/silica coatings for gravitational-wave detection Class. Quantum Grav. 24 405-15

[104] Robertson N A et al 2002 Quadruple suspension design for Advanced LIGO Class. Quantum Grav. 19 4043-58

[105] Heptonstall A, Cagnoli G, Hough J and Rowan S 2006 Characterisation of mechanical loss in synthetic fused silica ribbons Phys. Lett. A 354 353-9

[106] Abbott R et al 2002 Seismic isolation for advanced LIGO Class. Quantum Grav. 19 1591-7 